\title{
Dynamical mass generation in three-dimensional supersymmetric U(1) gauge field theory
}

\author{
A. Campbell-Smith and N. E. Mavromatos \\ Theoretical Physics, University of Oxford, 1 Keble Road, Oxford OX1 3NP, United Kingdom \\ and Theory Division, CERN, 1211-CH Geneva 23, Switzerland
}

(Received 26 April 1999; published 19 October 1999)

\begin{abstract}
We investigate and contrast the nonperturbative infrared structure of $\mathcal{N}=1$ and $\mathcal{N}=2$ supersymmetric noncompact $\mathrm{U}(1)$ gauge field theory in three space-time dimensions with $N$ matter flavors. We study the Dyson-Schwinger equations in a general gauge using the superfield formalism; this ensures that supersymmetry is kept manifest, though it leads to spurious infrared divergences which we have to avoid carefully. In the $\mathcal{N}=1$ case the superfield formalism allows us to choose a vertex which satisfies the U(1) Ward identity exactly, and we find the expected critical behavior in the wave function renormalization and strong evidence for the existence of a gauge independent dynamically generated mass, but with no evidence for a critical flavor number. We study the $\mathcal{N}=2$ model by dimensional reduction from four-dimensional $\mathcal{N}=1$ electrodynamics, and we refine the old gauge dependence argument that there is no dynamical mass generation. We recognize that the refinement only holds after dimensional reduction. [S0556-2821(99)09218-8]
\end{abstract}

PACS number(s): 11.30.Pb, 11.10.Kk, 11.15.Pg

\section{INTRODUCTION}

Dynamical mass generation is an interesting strongcoupling phenomenon which requires nonperturbative methods, and has been studied extensively in $\mathrm{N}$-flavor noncompact $\mathrm{U}(1)$ gauge theory in three dimensions $\left(\mathrm{QED}_{3}\right)$ [1-7]. In this nonsupersymmetric model the dynamics drives the coupling to a nontrivial fixed point in the infrared [6,7]: this leads to dynamical mass generation (and hence chiral symmetry breaking) and critical behavior in the normal (chirally symmetric) phase.

The situation for supersymmetric versions of electrodynamics is less clear. In an early work on three-dimensional $\mathcal{N}=2$ supersymmetric QED, it was argued using component formalism that (at least in Landau gauge) a dynamical mass is generated [1]. It was pointed out that the vanishing of the effective potential in supersymmetric field theories meant that the question of energetic favorability could not be answered simply; it was conjectured that the issue of the selection of the finite solution over the vanishing one might be resolved at the level of the effective action, and the Ward identities arising from the supersymmetry [1]. However, it has been argued on the grounds of gauge dependence that a (nonperturbative) nonrenormalization theorem in fourdimensional $\mathcal{N}=1$ supersymmetric electrodynamics forbids the dynamical generation of mass [8]. On dimensional reduction, four-dimensional $\mathcal{N}=1$ supersymmetry reduces to three dimensional $\mathcal{N}=2$ extended supersymmetry, and so the nonrenormalization theorem should also hold for the threedimensional extended model. Recently there has been speculation that the gauge dependence argument for the fourdimensional model is not sound $[9,10]$. The analysis of the four-dimensional model employs a simplified form of the U(1) Ward identity, and a heavily truncated vertex [8]. This is because in four dimensions supersymmetry and $\mathrm{U}(1)$ gauge invariance require, in addition to the usual three point vertex, $n$-point vertices for all $n>3$ (see Sec. VII). This problem does not arise in the three-dimensional $\mathcal{N}=1$ model, where supersymmetry and U(1) gauge invariance re- quire three- and four-point vertices only. Recently there has been evidence from numerical studies [11] that the $\mathcal{N}=2$ model does not generate a mass dynamically, in line with the dimensional reduction of the nonrenormalization theorem, but in contradistinction to the results of Ref. [1].

An early work on $\mathcal{N}=1$ supersymmetric QED in three dimensions demonstrated that troublesome logarithmic divergences present in the wave function renormalization of nonsupersymmetric $\mathrm{QED}_{3}$ are absent in the supersymmetric model, and it was argued that this allowed dynamical mass generation to take place for numbers of flavors less than a critical number [12], which was related to the critical flavor number of nonsupersymmetric $\mathrm{QED}_{3}$ [2]. The analysis was done in component formalism, in Landau gauge and with a trivial three-point vertex (set to unity) which does not satisfy the U(1) Ward identity except in the limits of zero transferred momentum and trivial (unit) wave function renormalization.

It is therefore of interest to look more closely at the infrared structure of both $\mathcal{N}=1$ and $\mathcal{N}=2$ supersymmetric $\mathrm{U}(1)$ gauge field theory. The actual dynamically generated mass is the $p \rightarrow 0$ limit of the mass function and is the pole of the matter two point correlation function; as such, it must be a gauge invariant object. Only if the $p \rightarrow 0$ limit leaves a gauge independent constant can it be said with certainty that a mass has been dynamically generated. It is therefore crucial to give a careful treatment of gauge dependence, and to look closely at the full vertex. Since supersymmetry cannot be dynamically broken in a supersymmetric U(1) gauge field theory [13], we do not have to consider the possibility of different dynamical masses for each component of the multiplet. With these issues in mind, we choose to work in superfield formalism, which keeps supersymmetry manifest, and we will, as far as possible, work in a general gauge. In components computations (see, e.g., Ref. [14]) it is necessary to use the U(1) Ward identity and the Ward identities arising from supersymmetry to constrain the vertices: the advantage of the superfield formalism is that we only need to examine the U(1) Ward identity, for the formalism ensures that the 
supersymmetry Ward identities are satisfied. In particular, by using the superfield formalism we will be able to choose a three-point vertex which satisfies the U(1) Ward identity exactly in the $\mathcal{N}=1$ case. It is known $[8,15]$ that superfield formalism for gauge theories results in spurious infrared divergences arising from the propagation of a gauge artifact: by careful choice of approximations and, where necessary, gauge, we can avoid most of the infrared divergences and they do not spoil our results.

We use the same approach to study the nonperturbative physics of both the $\mathcal{N}=1$ and $\mathcal{N}=2$ models: we construct a nonperturbative ansatz for the matter superfield propagator, and study the behavior of the functions appearing therein through the Dyson-Schwinger equations. In principle there are Dyson-Schwinger equations for the matter superfields, the gauge superfield, and for the full vertex. The gauge superfield Dyson-Schwinger equation will be resummed to leading order in $1 / N$, where $N$ is the number of matter flavors; this is exactly what is done in normal $\mathrm{QED}_{3}$. The vertex will be constrained by the U(1) Ward identity. When the results of the resummation and a choice for the vertex are put into the Dyson-Schwinger equation for the matter superfield, coupled integral equations for the self-energy and wave function renormalization result, which have to be solved. As with normal $\mathrm{QED}_{3}$, approximations have to be sought in order to look for solutions of the equations.

For the $\mathcal{N}=1$ model we will present two approaches for studying the Dyson-Schwinger equations. First, we consider some of the simplest approximations, which are sufficient when applied to normal $\mathrm{QED}_{3}$ to demonstrate the existence of dynamical mass generation (at least in Landau gauge) [3]. The advantages of making these computationally convenient approximations are twofold: they allow us to work in a general gauge throughout, and admit conversion of the integral equation to an equivalent differential equation. The differential equation is simple to solve, and has a gauge invariant, constant solution in the $p \rightarrow 0$ limit. The second method incorporates the full vertex, consistent with the U(1) Ward identity. Ideally we would like to probe the gauge dependence in exactly the same way again; however, the richer structure of the integral equations arising from this approach only admit more direct methods. In both approaches we find the expected critical behavior in the wave function renormalization [16-18]

$$
Z(p) \sim\left(\frac{p}{\alpha}\right)^{\gamma}, \quad \gamma \sim \mathcal{O}(1 / N),
$$

and exhibit the possibility of a finite dynamically generated mass. In contrast with Ref. [12] we find no evidence for a critical flavor number, above which there is no dynamical mass generation.

For the $\mathcal{N}=2$ model we again make some simple approximations which allow us to probe the gauge dependence. Again we find the expected critical behavior in the wave function renormalization (1.1), but this time we find that there is no gauge independent dynamically generated mass. This is based on a refinement of the gauge dependence argument of Ref. [8]; we note that the refinement relies on the existence of the scale arising from the compactified dimension, so that the nonrenormalization theorem for the fourdimensional model [8] may still be evaded $[9,10]$.

In Sec. II we construct the action functional for a $\mathcal{N}=1$ supersymmetric theory of $N$ matter flavors interacting with a U(1) gauge field in superfield formalism. We also give the dressed propagators for the model which result from this action. We construct the U(1) Ward identity in Sec. III using the Becchi-Rouet-Stora (BRS) method, and in Sec. IV we construct the Dyson-Schwinger equations for the model, which we use to analyze the nonperturbative properties of the theory. We present and briefly discuss our simple computation in Sec. V, in which we are able to probe the gauge dependence of the mass function by making convenient approximations in the integral equations. Our more complete computation follows in Sec. VI, where we choose a vertex which satisfies the U(1) Ward identity. Here we cannot use the elegant methods of Sec. V, but we are able to demonstrate that the solution obtained there persists in this more complete computation. To conclude this part of the paper, we also discuss the question of whether the finite mass solution is preferred to the vanishing mass solution, in the context of the suggestion [1] that this can be answered by appealing to the effective action and the supersymmetry Ward identities. We find that no extra information can be obtained in this way, in contrast to the models of Refs. [19-21] which, crucially, have extra constraints.

We turn to the $\mathcal{N}=2$ extended model in Sec. VII, where we build an action functional and dressed propagators for four-dimensional $\mathcal{N}=1$ supersymmetric $N$-flavor electrodynamics. We construct the Dyson-Schwinger equations for the model in Sec. VIII and then compactify one dimension to obtain the coupled integral equations for the threedimensional $\mathcal{N}=2$ model we wish to analyze. We compute the wave function renormalization and refine the argument of Ref. [8] for the nonexistence of dynamical mass generation.

We append discussion and concluding remarks in Sec. IX, and briefly contrast with the situation in nonsupersymmetric $\mathrm{QED}_{3}$. In the appendixes we collect some useful results and basic features of $\mathcal{N}=1$ supersymmetry in three and four dimensions, which are essential to the analysis of the models we consider.

\section{THE $\mathcal{N}=1$ ACTION}

The $\mathcal{N}=1$ rigid superspace in three dimensions can be parametrized by the usual three space-time coordinates and two Grassmann-odd coordinates arranged in the Majorana spinor $\theta^{\alpha}$, where $\alpha \in\{1,2\}$ [22], which we denote collectively by the symbol $z$. We collect some basic features of this $\mathcal{N}=1$ superspace and superfields in Appendix A. Consider complex scalar "matter" superfields $\Phi, \Phi^{*}$ (mappings from superspace to $\mathrm{C}$ ) which transform under local $\mathrm{U}(1)$ transformations in the familiar way

$$
\begin{gathered}
\Phi(z) \rightarrow \Phi^{\prime}\left(z^{\prime}\right)=e^{+i e G(z)} \Phi(z), \\
\Phi^{*}(z) \rightarrow \Phi^{* \prime}\left(z^{\prime}\right)=\Phi^{*}(z) e^{-i e G(z)} .
\end{gathered}
$$


Here $e$ is the (dimensionful) gauge coupling and $G(z)$ must be a real scalar superfield (a mapping from superspace to $R$ ) to preserve the superfield nature of $\Phi^{\prime}$; in order to build an action functional invariant under local U(1) transformations, we must construct covariant derivatives which transform in the same way as the matter superfields themselves:

$$
\nabla_{\alpha} \rightarrow \nabla_{\alpha}^{\prime}=e^{i e G(z)} \nabla_{\alpha} e^{-i e G(z)} .
$$

The covariant derivatives can be written in terms of a real spinor (superfield) connection $\Gamma_{\alpha}$ as follows:

$$
\nabla_{\alpha}=D_{\alpha}-i e \Gamma_{\alpha} ;
$$

here $D_{\alpha}$ is the normal spinorial derivative, covariant with respect to supersymmetry transformations [22]. The connection transforms in the usual way under infinitesimal local $\mathrm{U}(1)$ transformations

$$
\Gamma_{\alpha}(z) \rightarrow \Gamma_{\alpha}^{\prime}\left(z^{\prime}\right)=\Gamma_{\alpha}(z)+D_{\alpha} G(z) .
$$

We wish to consider a model with $\mathcal{N}=1$ supersymmetry, local U(1) gauge invariance and $N$ matter flavors. The required action then comprises three parts: the gauge invariant classical field strength term for the connection $\Gamma_{\alpha}$, a (Lorentz) gauge fixing term and a locally $\mathrm{U}(1)$ invariant kinetic term for the matter superfields $\Phi$ and $\Phi^{*}$ :

$$
\begin{gathered}
S=S_{g}^{\text {class }}+S_{g}^{\mathrm{GF}}+S_{m} ; \\
S_{g}^{\text {class }}=\int d^{3} x d^{2} \theta \Gamma_{\alpha}\left(-\frac{1}{8} D^{\eta} D^{\alpha} D^{\beta} D_{\eta}\right) \Gamma_{\beta}, \\
S_{g}^{\mathrm{GF}}=\int d^{3} x d^{2} \theta \Gamma_{\alpha}\left(\frac{1}{4 \xi} D^{\alpha} D^{2} D^{\beta}\right) \Gamma_{\beta}, \\
S_{m}=\int d^{3} x d^{2} \theta\left(-\frac{1}{2}\right)\left[\nabla^{\alpha} \Phi\right]^{*}\left[\nabla_{\alpha} \Phi\right] .
\end{gathered}
$$

We have included in the matter part an implicit sum over $N$ flavors, which do not interact with each other but interact with the same gauge field. The parameter $\xi$ is the familiar gauge parameter; supersymmetric Feynman and Landau gauges are given by $\xi=1,0$ respectively. From this action it is easy to derive the renormalized propagators for the matter fields and connection

$$
\begin{gathered}
\Delta(p ; 12)=i \frac{Z(p) D^{2}-\Sigma(p)}{Z^{2}(p) p^{2}+\Sigma^{2}(p)} \delta^{2}(12), \\
\Delta_{\alpha \beta}(p ; 12)=-i \frac{1}{p^{4}} \frac{1}{1+\alpha /|p|} \\
\times\left[(1+\xi) p_{\alpha \beta} D^{2}-(1-\xi) C_{\alpha \beta} p^{2}\right] \delta^{2}(12) .
\end{gathered}
$$

The gauge field propagator includes the effects of massless matter loops (to leading order in $1 / N$ ) in the vacuum polarization factor [1]

$$
(1+\alpha /|p|)^{-1} .
$$

Both the spinor and scalar components of the matter superfield $\Phi$ contribute to this correction, and so the vacuum polarization is exactly twice that of nonsupersymmetric $\mathrm{U}(1)$ gauge theory [1,12], and the effective ultraviolet scale $\alpha$ is given by

$$
\alpha \doteq \frac{e^{2} N}{4} .
$$

The second rank antisymmetric symbol $C_{\alpha \beta}$ appearing in the gauge field propagator acts as a metric for spinor indices [22] (see Appendix A) and we have written three vectors in a convenient spinor notation: $p_{\alpha \beta}$ is a symmetric second rank spinor. In all the above an abbreviated superspace notation has been used where, for instance,

$$
\delta^{2}(12) \equiv \delta^{2}\left(\theta_{1}-\theta_{2}\right) \doteq-\left(\theta_{1}-\theta_{2}\right)^{2} .
$$

The interaction piece of the action is given by

$$
\begin{aligned}
S_{\mathrm{int}}= & \int d^{3} x d^{2} \theta\left(-\frac{1}{2} C^{\alpha \beta} i e \Gamma_{\alpha} \Phi D_{\beta} \Phi^{*}\right. \\
& \left.+\frac{1}{2} C^{\alpha \beta} i e \Gamma_{\alpha} \Phi^{*} D_{\beta} \Phi-\frac{1}{2} e^{2} C^{\alpha \beta} \Gamma_{\beta} \Gamma_{\alpha} \Phi^{*} \Phi\right) .
\end{aligned}
$$

The Feynmann rules derived from this action are given at the end of Appendix A. Finally, note that we have not used any Wess-Zumino type gauge fixing for the connexion superfield; this is not a gauge invariant truncation (nor one which respects supersymmetry), and we wish, as far as is possible, to investigate the full gauge dependence of the nonperturbative correlation functions.

\section{BRS INVARIANCE AND WARD IDENTITIES}

Following the BRS approach, to compute the Ward identities for this model we promote the gauge fixed action (2.5) to an action invariant under an enhanced gauge symmetry. The gauge fixing term in Eq. (2.5) transforms under infinitesimal gauge transformations as follows:

$$
\delta_{\mathrm{G}} \frac{1}{4 \xi} \Gamma_{\alpha}\left(D^{\alpha} D^{2} D^{\beta}\right) \Gamma_{\beta}=\frac{1}{\xi} G\left(\square D^{\alpha} \Gamma_{\alpha}\right) .
$$

To make a BRS invariant action, we add a ghost term to the action, whose transformation properties cancel those of the gauge fixing term

$$
S^{\text {ghost }}=\int d^{3} x d^{2} \theta(-u \square w),
$$

where $u, w$ are real Grassmann-odd scalar ghost superfields. The full action is then invariant under the extended gauge symmetry

$$
\delta_{\eta} \Phi=i e(\eta w) \Phi
$$




$$
\begin{gathered}
\delta_{\eta} \Phi^{*}=-i e \Phi^{*}(\eta w), \\
\delta_{\eta} \Gamma_{\alpha}=D_{\alpha}(\eta w), \\
\delta_{\eta} u=-\frac{1}{\xi} \eta D^{\alpha} \Gamma_{\alpha}, \\
\delta_{\eta} w=0 ;
\end{gathered}
$$

here $\eta$ is a real Grassmann-odd number. Note that $\delta_{\eta}^{2}=0$. The generating functional can now be written

$$
\mathcal{Z}=\int \mathbb{D} \Phi \mathbb{D} \Phi^{*} \mathrm{D} \Gamma_{\alpha} \mathrm{D} u \mathbb{D} w e^{i S+i S_{\mathrm{s}}-i \Delta_{\mathrm{s}}},
$$

where

$$
\begin{aligned}
S_{\mathrm{s}} & =\int d^{3} x d^{2} \theta\left(J^{*} \Phi+J \Phi^{*}+K^{\alpha} \Gamma_{\alpha}+\sigma u+\tau w\right), \\
\Delta_{\mathrm{s}}= & \int d^{3} x d^{2} \theta\left(J^{*} \delta_{\eta} \Phi+J \delta_{\eta} \Phi^{*}+K^{\alpha} \delta_{\eta} \Gamma_{\alpha}+\sigma \delta_{\eta} u\right. \\
& \left.+\tau \delta_{\eta} w\right) .
\end{aligned}
$$

The set $\left\{J, J^{*}, K^{\alpha}, \sigma, \tau\right\}$ are superfield sources; the last three are Grassmann-odd valued and $K$ is a spinor. Using the fact that $\eta^{2}=0$, the Ward identity immediately follows:

$$
\begin{aligned}
0= & \int \mathrm{D} \Phi \mathrm{D} \Phi^{*} \mathrm{D} \Gamma_{\alpha} \mathrm{D} u \mathrm{D} w e^{i S+i S_{\mathrm{s}}}\left(\int d ^ { 3 } x d ^ { 2 } \theta \left(J^{*} \delta_{\eta} \Phi\right.\right. \\
& \left.\left.+J \delta_{\eta} \Phi^{*}+K^{\alpha} \delta_{\eta} \Gamma_{\alpha}+\sigma \delta_{\eta} u+\tau \delta_{\eta} w\right)\right)
\end{aligned}
$$

To interpret this in terms of correlation functions for the model, we construct the quantum effective action (the index $c$ indicates a classical value):

$$
\begin{aligned}
\Gamma\left[\Phi_{c},\right. & \left.\Phi_{c}^{*}, \Gamma_{\alpha}^{c}, u_{c}, w_{c}\right] \\
= & -i \ln \mathcal{Z}\left[J, J^{*}, K^{\alpha}, \sigma, \tau\right] \\
& -\int d^{3} x d^{2} \theta\left(J \Phi_{c}^{*}+J^{*} \Phi_{c}+K^{\alpha} \Gamma_{\alpha}^{c}+\sigma u_{c}+\tau w_{c}\right)
\end{aligned}
$$

the sources are given by functional derivatives of the effective action

$$
\begin{gathered}
\frac{\delta \Gamma}{\delta \Phi_{c}}=-J^{*}, \quad \frac{\delta \Gamma}{\delta \Phi_{c}^{*}}=-J, \\
\frac{\delta \Gamma}{\delta \Gamma_{\alpha}^{c}}=K^{\alpha}, \\
\frac{\delta \Gamma}{\delta u_{c}}=\sigma, \quad \frac{\delta \Gamma}{\delta w_{c}}=\tau,
\end{gathered}
$$

$$
\begin{aligned}
\Gamma\left[\Phi_{c},\right. & \left.\Phi_{c}^{*}, \Gamma_{\alpha}^{c}, u_{c}, w_{c}\right] \\
= & \int d^{5} x d^{5} y\left\{\Phi_{c}^{*}(x) \Delta^{-1}(x-y) \Phi_{c}(y)+\Gamma_{\alpha}(x)\right. \\
& \left.\times\left[\Delta^{-1}(x-y)\right]^{\alpha \beta} \Gamma_{\beta}(y)\right\} \\
& +\int d^{5} x d^{5} y d^{5} z\left(\Phi_{c}^{*}(x) \Gamma_{\alpha}^{c}(y) \frac{e}{2} G(x, y, z) D^{\alpha} \Phi_{c}(z)\right. \\
& \left.+\Phi_{c}(x) \Gamma_{\alpha}^{c}(y) \frac{e}{2} G(x, y, z) D^{\alpha} \Phi_{c}^{*}(z)\right)+\cdots
\end{aligned}
$$

Now we can carry out the functional integral in the identity (3.6) to obtain

$$
\begin{aligned}
0= & \int d^{5} x\left(i e \frac{\delta \Gamma}{\delta \Phi_{c}^{*}} \Phi_{c}^{*} w_{c}-i e \frac{\delta \Gamma}{\delta \Phi_{c}} w_{c} \Phi_{c}+D_{\alpha} \frac{\delta \Gamma}{\delta \Gamma_{\alpha}^{c}} w_{c}\right. \\
& \left.+\frac{1}{\xi} \frac{\delta \Gamma}{\delta u_{c}} D^{\alpha} \Gamma_{\alpha}^{c}\right) .
\end{aligned}
$$

Taking functional derivatives with respect to $w_{c}, \Phi_{c}^{*}$ and $\Phi_{c}$ we obtain the Ward identity in configuration space:

$$
\begin{aligned}
0= & D^{2}(y) G(x, y, z)+i \Delta^{-1}(x-z) \delta(x-y) \\
& -i \Delta^{-1}(x-z) \delta(z-y)
\end{aligned}
$$

which in momentum space reads

$$
D^{2}(p-q) G(p, p-q, q)=i \Delta^{-1}(p)-i \Delta^{-1}(q) \text {. }
$$

The advantage of using a superspace formulation is now apparent, for using the identity

$$
\left[D^{2}(p)\right]^{2}=-p^{2}
$$

the Ward identity (3.12) can be inverted. In fact, as we shall see, the structure of the Dyson-Schwinger equations is such that it can always be organized so that $D^{2}(p-q)$ acts to the left of the full vertex, and so the Ward identity can be used exactly as it appears in Eq. (3.12). Of course, as with the nonsupersymmetric U(1) theory, the Ward identity does not constrain the transverse part of the vertex, for which

$$
D^{2}(p-q) G(p, p-q, q)=0 .
$$

Since, as discussed above, the integral equations can be arranged so that the full vertex only appears with an accompanying $D^{2}$, this will not be a problem for our analysis. Moreover, in not having to invert the Ward identity, we will not introduce any extra kinematical singularities as $p \rightarrow q$; this is known to cause problems in the nonsupersymmetric model [23].

In principle the four-point vertex could also be constrained by the U(1) Ward identity; however, the form depends in a complicated way on the three-point vertex and it is sufficient for us to choose a trivial function for our fourpoint vertex. As we will show, the graph which includes the 
four-point vertex contributes only to the wave function renormalization, and the contribution vanishes in supersymmetric Feynman gauge.

\section{THE DYSON-SCHWINGER EQUATIONS}

The nonperturbative properties of the model are determined through the Dyson-Schwinger equations. These equations lead to coupled integral equations for the self-energy and wave function renormalization, which can be solved (in principle) to yield the dynamically generated mass

$$
M \doteq \lim _{p \rightarrow 0} M(p) \equiv \lim _{p \rightarrow 0} \frac{\Sigma(p)}{Z(p)}
$$

The truncated Dyson-Schwinger equation we will use is

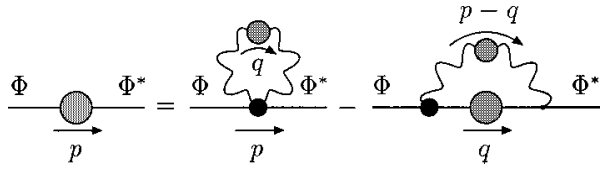

FIG. 1. Schematic form of the Dyson-Schwinger equation for the nonperturbative matter two point correlation function. Solid lines represent matter superfield propagators, and wavy lines gauge superfield propagators; blobs indicate full nonperturbative quantities.

shown graphically in Fig. 1. The graph on the left hand side is a convenient shorthand for the difference between the full inverse propagator and the bare inverse propagator.

The graph on the left hand side of Fig. 1 is given by

$$
\begin{aligned}
& -i \int d^{2} \theta_{1} d^{2} \theta_{2} \Phi\left(-p, \theta_{1}\right)\left[Z(p) D^{2}(p)+\Sigma(p)\right] \delta^{2}(12) \Phi^{*}\left(p, \theta_{2}\right)+i \int d^{2} \theta \Phi(-p, \theta) D^{2}(p) \Phi^{*}(p, \theta) \\
& \quad=-i[Z(p)-1] \int d^{2} \theta \Phi(-p, \theta) D^{2}(p) \Phi^{*}(p, \theta)-i \Sigma(p) \int d^{2} \theta \Phi(-p, \theta) \Phi^{*}(p, \theta) .
\end{aligned}
$$

The first "seagull" graph on the right is simply

$$
\int \frac{d^{3} q}{(2 \pi)^{3}} d^{2} \theta_{1} d^{2} \theta_{2} \Phi\left(-p, \theta_{1}\right)\left[-\frac{e^{2}}{2} G_{4} C^{\beta \alpha} \delta^{2}(12) \Delta_{\alpha \beta}(P ; 12)\right] \Phi *\left(p, \theta_{2}\right),
$$

and the last graph is given by $(P \doteq p-q)$

$$
\int \frac{d^{3} q}{(2 \pi)^{3}} d^{2} \theta_{1} d^{2} \theta_{2} \Phi\left(-p, \theta_{1}\right)\left[-\frac{e^{2}}{4} G_{3}(p, P, q) D^{\alpha}(q) D^{\beta}(q) \Delta(q ; 12)\right] \Delta_{\alpha \beta}(P ; 12) \Phi *\left(p, \theta_{2}\right) .
$$

Our use of a symmetric second rank spinor notation for three vectors immediately implies the following identities:

$$
\begin{gathered}
C^{\alpha \beta} p_{\alpha \beta}=0, \\
q^{\alpha \mu} p_{\mu \beta}=\delta_{\beta}^{\alpha} p \cdot q .
\end{gathered}
$$

Note that the second of these identities indicates that the $C$ symbol from the four-point vertex in the last graph projects out only the $(1-\xi)$ component of the gauge field propagator, and indeed the entire graph would vanish in the supersymmetric Feynman gauge $\xi=1$.

Upon substitution of the propagators (2.7), the superspace integrations can be performed using the identities above along with the relations (A1) and (A4) in Appendix A. We will present two computations in what follows: first, a computation in which the simplest approximations are made: $G_{3}(p, P, q)$ is set to $Z(q)$, and the bifurcation method is used, in which $\Sigma$ is set to vanish in the denominators of all the kernels. These approximations are drastic but computationally extremely convenient, and are sufficient to demonstrate the existence of a dynamically generated mass in non- supersymmetric U(1) gauge theory [3]. These approximations will give us enough flexibility to probe the gauge dependence of the solution. Second, we present a more complete computation, in which the full three-vertex consistent with the Ward identity (3.12) is used.

The treatments in the two cases follow a similar strategy: inserting the propagators (2.7) into Eqs. (4.4) and (4.3) along with a choice for the three-point vertex, the superspace parts of the integrals can be unpacked and reassembled using the identities (4.5), (A1), and (A4) to appear as functions of $\{Z, \Sigma, p, q\}$ multiplying the two superspace structures

$$
\begin{gathered}
\int d^{2} \theta \Phi(-p, \theta) D^{2}(p) \Phi^{*}(p, \theta) \\
\int d^{2} \theta \Phi(-p, \theta) \Phi^{*}(p, \theta) .
\end{gathered}
$$

Comparison with Eq. (4.2) shows that the function multiplying the first of these structures is to be identified with the wave function renormalization, and that multiplying the second with the self-energy function. From this point the usual 
procedure followed with the nonsupersymmetric model can be adopted to study the coupled integral equations which multiply the structures above. The angular integrations can be performed, leaving integral equations now over the variable $|q|$ only, which can in principle be evaluated. In practice, the kernels have to be approximated with lowmomentum expansions. This is justified, for the (supersymmetric and nonsupersymmetric) model is superrenormalizable, and dynamical mass generation, if it occurs at all, occurs in the deep infrared. Two methods of solution can be employed for the resulting approximate integral equations: conversion to an equivalent differential equation, or direct integration with trial functions. The first of these will be used with our simple approximation, and the second for the more complete computation.

\section{SIMPLE COMPUTATION: GAUGE DEPENDENCE}

In this section we present a computation of the wave function renormalization and self-energy functions based on the simplest approximations. In defense of the approximations, they are sufficient to demonstrate the existence of a dynamically generated mass in nonsupersymmetric $\mathrm{U}(1)$ gauge theory [3]; the three-point vertex we choose is compatible with the Ward identity only in the limit of vanishing transferred momentum $(p)$, though it is consistent with a nontrivial wave function renormalization. The results of the computation for our model are clear: the expected critical behavior [16-18] is found for the wave function renormalization, and there is a simple form for a gauge independent dynamically generated mass. This is shown in a neat way, by converting the integral equation for the mass function to an equivalent differential equation, from which it is obvious that a $\xi$-independent constant solution exists. It is impossible to use this neat method in the more complete computation to follow in Sec. VI, for the rich structure of the integral equation resulting from the full vertex prohibits the conversion to a differential equation.

In the approximation $G_{3}=Z(q)$ the superspace parts of the integrals can be unpacked as described above, yielding the following integral equations for the wave function renormalization and self-energy function $\left[\mathbb{K}(q) \doteq Z^{2}(q) q^{2}\right.$ $\left.+\Sigma^{2}(q)\right]$ :

$$
\begin{aligned}
Z(p)=1 & +\frac{1}{4} \frac{\alpha}{N \pi^{2}} \int d q q^{2} \frac{Z^{2}(q)}{\mathbb{K}(q)} \int d x \frac{1}{P^{4}} \frac{1}{1+\alpha /|P|} 2(1+\xi) \\
& \times\left(q p x-q^{2}\right), \\
\Sigma(p)= & \frac{1}{4} \frac{\alpha}{N \pi^{2}} \int d q q^{2} \frac{\Sigma(q) Z(q)}{\mathbb{K}(q)} \int d x \frac{1}{P^{4}} \frac{1}{1+\alpha /|P|} \\
& \times\left[2(1+\xi)\left(q p x-q^{2}\right)+2(1-\xi) P^{2}\right],
\end{aligned}
$$

where $P^{2}=p^{2}+q^{2}-2 p q x$. The seagull graph (4.3) gives only an irrelevant $p$ independent contribution to $Z$ which would vanish in the supersymmetric Feynmann gauge, and does not contribute at all to $\Sigma$ :

$$
Z_{\text {seagull }} \simeq \frac{(1-\xi)}{N \pi^{2}} \int d q\left(\frac{1}{q}-\frac{1}{q+\alpha}\right)
$$

The angular $x$ integrations in Eq. (5.1) can be performed easily to obtain

$$
\begin{aligned}
Z(p)=1 & +\frac{1}{4} \frac{\alpha}{N \pi^{2}} \int d q 2 q^{2} \frac{Z^{2}(q)(1+\xi)}{\mathbb{K}(q)} \mathcal{I}_{1}(p, q ; \alpha), \\
\Sigma(p)= & \frac{1}{4} \frac{\alpha}{N \pi^{2}} \int d q 2 q^{2} \frac{Z(q) \Sigma(q)}{\mathbb{K}(q)} \\
& \times\left[(1+\xi) \mathcal{I}_{1}(p, q ; \alpha)+(1-\xi) \mathcal{I}_{2}(p, q ; \alpha)\right],
\end{aligned}
$$

where the kernels $\mathcal{I}_{i}$ are given by

$$
\begin{gathered}
\mathcal{I}_{1}=\frac{1}{2 p q}\left(\frac{p^{2}-q^{2}}{\alpha}\left[\frac{1}{|p-q|}-\frac{1}{p+q}\right]-\frac{p^{2}-q^{2}}{\alpha^{2}} \ln \left[\frac{p+q}{|p-q|}\right]\right. \\
\left.+\frac{p^{2}-q^{2}-\alpha^{2}}{\alpha^{2}} \ln \left[\frac{p+q+\alpha}{|p-q|+\alpha}\right]\right), \\
\mathcal{I}_{2}=\frac{1}{p q} \ln \left[\frac{p+q+\alpha}{|p-q|+\alpha}\right] .
\end{gathered}
$$

In the limit of $p, q \ll \alpha$ the logarithms can be expanded

$$
\ln \left[\frac{p+q+\alpha}{|p-q|+\alpha}\right] \simeq \theta(p-q)\left(\frac{2 q}{\alpha}-2 \frac{p q}{\alpha^{2}}+\cdots\right)+\langle p \leftrightarrow q\rangle,
$$

and upon using the bifurcation method in the denominators the gap equations take on the following approximate form:

$$
\begin{gathered}
Z(p)=1+\frac{1}{2} \frac{(1+\xi)}{N \pi^{2} \alpha p^{2}} \int_{0}^{p} d q q^{2} \\
-\frac{1}{2} \frac{(1+\xi)}{N \pi^{2} \alpha} \int_{p}^{\alpha} d q\left(2-\frac{2 \alpha}{q}-\frac{p^{2}}{q^{2}}\right), \\
\Sigma(p)=\frac{(1-\xi)}{N \pi^{2}} \int_{0}^{p} d q \frac{1}{p} \frac{\Sigma(q)}{Z(q)}-2 \frac{\xi}{N \pi^{2}} \int_{p}^{\alpha} d q \frac{1}{q} \frac{\Sigma(q)}{Z(q)} .
\end{gathered}
$$

\section{A. Wave function renormalization}

The first of equations (5.5) can be integrated directly, to obtain the wave function renormalization in the normal phase (where there is no mass generation) and as an approximation to the wave function renormalization when there is mass generation:

$$
Z(p) \simeq a+\left(\frac{p}{\alpha}\right)^{\gamma}+f\left(\frac{p}{\alpha}\right),
$$


where $a$ is a constant and the function $f$ vanishes at least as quickly as $p / \alpha$ in the limit $p \rightarrow 0$. In the above we have used the usual renormalization group argument to resum logarithmic terms as

$$
1+\gamma \ln \left(\frac{p}{\alpha}\right) \simeq\left(\frac{p}{\alpha}\right)^{\gamma} .
$$

The exponent $\gamma$ is given by

$$
\gamma \simeq(1+\xi) \frac{1}{N \pi^{2}} .
$$

This critical behavior is qualitatively the same as that found in nonsupersymmetric electrodynamics $[17,18]$, so this gives us confidence in our approach, and demonstrates that the rough qualitative behavior is exhibited even within the drastic approximation scheme used here. The nontrivial wave function renormalization we have found here is to be contrasted with the early results of Ref. [12] in which the wave function renormalization was taken to be unity.

\section{B. Self-energy}

Turning now to the self-energy function, we wish to construct an equivalent differential equation for the mass function

$$
M(p ; \xi)=\frac{\Sigma(p ; \xi)}{Z(p ; \xi)} ;
$$

this is achieved by differentiating the second integral equation (5.5) twice with respect to $p$ and once with respect to $\xi$. The reason we take an extra derivative with respect to $\xi$ is that this will allow us to probe the gauge dependence of the solution directly, without having to fully solve the equation, for we are only interested in the gauge dependence of the solution. First we rescale variables as $p \mapsto \alpha p$ and $M(p) \mapsto \alpha m(p)$ and then the resulting differential equation is

$$
\begin{aligned}
(\dot{m})^{\prime \prime} & +\frac{1}{p}\left(2-\frac{1}{N \pi^{2}}(1+\xi)\right)(\dot{m})^{\prime}-\frac{2}{N \pi^{2}} \frac{\xi}{p^{2}} \dot{m}-\frac{1}{N \pi^{2}} \frac{1}{p} m^{\prime} \\
& -\frac{2}{N \pi^{2}} m=0
\end{aligned}
$$

the superior point denotes a derivative with respect to $\xi$ and primes denote derivatives with respect to $p$.

Demanding that $\dot{m}=0$ for all values of $p$ (in the infrared) yields the solution

$$
M(p)=M_{0} e^{-p^{2} / \alpha^{2}},
$$

which is obviously constant and $\xi$ independent in the $p \rightarrow 0$ limit. We should be cautious, however, for the approximation of neglecting the mass function in the denominators might not be consistent with this limit. When, in the next section, we use the full vertex and restore the mass in the denominators, we will be able to show that this solution ap- pears to persist in the limit $p \rightarrow 0$, confirming our assertion here. It is interesting to note that there are no solutions to Eq. (5.8) which are gauge independent in the limit $p \rightarrow 0$ but gauge dependent elsewhere (this can be shown by developing a gauge dependent power series for $m$ and then constraining the gauge dependence via the indicial equation): this contrasts the case of nonsupersymmetric $\mathrm{QED}_{3}$, in which such solutions do appear to exist, and seem to lead to a critical flavor number. We will return to a full discussion of this issue in Sec. IX.

\section{Concluding remarks}

The approximations we have used in this section are rather drastic, but the results obtained are neat and transparent: the wave function renormalization exhibits the expected critical behavior [16-18]

$$
Z(p) \simeq\left(\frac{p}{\alpha}\right)^{\gamma}, \quad \gamma \sim \frac{1}{N},
$$

and we find a gauge independent solution for the dynamically generated mass

$$
M(p)=M_{0} e^{-p^{2} / \alpha^{2}} .
$$

These results are in line with those found in nonsupersymmetric $\mathrm{QED}_{3}$, though we have no evidence here for a critical flavor number above which no mass generation occurs, which is the case in the nonsupersymmetric model. This contrasts the results of Ref. [12], in which a critical flavor number was found. We discuss this issue further in Sec. IX after we have presented our full computation.

Had we not used the bifurcation method in this section, the resulting differential equation for the mass function would have been nonlinear. What is curious is that the bifurcation method works so well: as we will show, the solutions derived above are entirely consistent with the full vertex and the restoration of the mass in the denominators of the kernel. The inaccuracies arising from regions of integration where the bifurcation method is least reliable are suppressed in the limits considered for the determination of the dynamical mass (i.e., vanishing $p$ ).

\section{FULL COMPUTATION}

In this section we turn to our more complete computation, where we use a three-point vertex consistent with the $\mathrm{U}(1)$ Ward identity (3.12). The trick we use is to rewrite $P^{2}$ $=-D^{4}(P)$ in the gauge field propagator, and then integrate by parts until the $D^{2}(P)$ operators appear acting on the three-point vertex $G_{3}$, so that the Ward identity (3.12) can be used as it appears. When this is done, the superspace parts of the integral equations can be unpacked as described in Sec. IV and reconstructed after some algebra to obtain the coupled integral equations (in which we have already performed the angular integrations) 


$$
\begin{aligned}
Z(p)=1 & +\frac{1}{4} \frac{\alpha}{N \pi^{2}} \int d q \frac{q^{2}}{\mathbb{K}(q)}\left\{4(1+\xi) Z(p) Z(q) \mathcal{J}_{1}(p, q ; \alpha)\right. \\
+ & 2(1-\xi)\left[q^{2} Z(p) Z(q)-q^{2} Z^{2}(q)+\Sigma(p) \Sigma(q)\right. \\
& \left.\left.-\Sigma^{2}(q)\right] \mathcal{J}_{2}(p, q ; \alpha)\right\}, \\
\Sigma(p)= & \frac{1}{4} \frac{\alpha}{N \pi^{2}} \int d q \frac{q^{2}}{\mathbb{K}(q)}\{2(1+\xi)[\Sigma(p) Z(q) \\
& -\Sigma(q) Z(p)] \mathcal{J}_{1}(p, q ; \alpha)+2(1-\xi)\left[q^{2} \Sigma(p) Z(q)\right. \\
& \left.\left.-p^{2} \Sigma(q) Z(p)\right] \mathcal{J}_{2}(p, q ; \alpha)\right\} ;
\end{aligned}
$$

the angular integrals $\mathcal{J}_{i}(p, q ; \alpha)$ are given by

$$
\begin{gathered}
\mathcal{J}_{1}(p, q ; \alpha)=\frac{1}{2 p q}\left(\frac{p^{2}-q^{2}}{\alpha}\left[\frac{1}{|p-q|}-\frac{1}{p+q}\right]\right. \\
-\frac{p^{2}-q^{2}}{\alpha^{2}} \ln \left[\frac{p+q}{|p-q|}\right] \\
\left.+\frac{p^{2}-q^{2}-\alpha^{2}}{\alpha^{2}} \ln \left[\frac{p+q+\alpha}{|p-q|+\alpha}\right]\right), \\
\mathcal{J}_{2}(p, q ; \alpha)=\frac{1}{p q}\left(\frac{1}{\alpha}\left[\frac{1}{|p-q|}-\frac{1}{p+q}\right]-\frac{1}{\alpha^{2}} \ln \left[\frac{p+q}{|p-q|}\right]\right. \\
\left.+\frac{1}{\alpha^{2}} \ln \left[\frac{p+q+\alpha}{|p-q|+\alpha}\right]\right) .
\end{gathered}
$$

Again, the seagull graph gives an irrelevant $p$ independent contribution to $Z$, Eq. (5.2), and also vanishes in the supersymmetric Feynman gauge. Since much of our final analysis will have to be restricted to this gauge (or at least to approximations equivalent to considering this gauge), even if we were to make a $p$-dependent ansatz for $G_{4}$, the contribution would vanish on account of the $C^{\alpha \beta}$ tensor structure in the vertex projecting out only the $(1-\xi)$ component of the gauge field propagator.

To derive the integral equations we have used the following Taylor expansion for operators of the form $D^{2}(p+r)$ when acting on $\Phi^{*}(p, \theta)$ :

$$
\begin{aligned}
D^{2}(p+r)= & D^{2}(p)+\frac{1}{2} r^{\alpha \beta}\left[\frac{\partial}{\partial r^{\alpha \beta}} D^{2}(r)\right]_{r=p} \\
& +\frac{1}{2}\left[\frac{1}{2} \frac{\partial^{2}}{\partial r_{\alpha \beta} \partial r^{\alpha \beta}} D^{2}(r)\right]_{r=p} .
\end{aligned}
$$

Note that this is an exact expansion, which terminates at second order. The curious extra factors of one half which appear in the expansion arise on account of the second of Eqs. (4.5) which puts a factor of 2 into the dot product of two three vectors. When acting on $\Phi^{*}(p, \theta)$ we drop terms which involve derivatives with respect to $r$, for these corre- spond to higher derivative terms, which should be irrelevant for our study of the infrared physics.

To study the integral equations it is again necessary to approximate the logarithms with small momentum expansions, and we obtain approximate integral equations for $\Sigma$ and $Z$, in which we have used the abbreviated notations

$$
\begin{aligned}
A & \doteq \frac{1}{4} \frac{1}{N \pi^{2}}, \\
Q(p, q ; Z, \Sigma) & \doteq q^{2} Z(q)[Z(p)-Z(q)]+\Sigma(q)[\Sigma(p)-\Sigma(q)] \\
R(Z, \Sigma) & \doteq \Sigma(p) Z(q)-\Sigma(q) Z(p), \\
S(p, q ; Z, \Sigma) & \doteq q^{2} \Sigma(p) Z(q)-p^{2} \Sigma(q), Z(p) .
\end{aligned}
$$

The wave function renormalization is given by

$$
\begin{aligned}
Z(p)= & +\frac{4(1+\xi)}{\alpha p^{2}} A \int_{0}^{p} d q \frac{q^{4} Z(p) Z(q)}{\mathbb{K}(q)} \\
& +4(1+\xi) A \int_{p}^{\alpha} d q \frac{Z(p) Z(q)}{\mathbb{K}(q)}\left(\frac{2 q^{2}}{\alpha}-2 q-\frac{p^{2}}{\alpha}\right) \\
& +2(1-\xi) A \int_{0}^{p} d q \frac{Q(p, q ; Z, \Sigma)}{\mathbb{K}(q)}\left(\frac{q}{p^{2}-q^{2}}-\frac{2 q^{2}}{\alpha p^{2}}\right) \\
& +2(1-\xi) A \int_{p}^{\alpha} d q \frac{Q(p, q ; Z, \Sigma)}{\mathbb{K}(q)}\left(\frac{q^{2}}{p} \frac{1}{q^{2}-p^{2}}-\frac{2}{\alpha}\right),
\end{aligned}
$$

and for the self-energy we obtain

$$
\begin{aligned}
\Sigma(p)= & \frac{2(1+\xi)}{\alpha p^{2}} A \int_{0}^{p} d q \frac{q^{4} R(Z, \Sigma)}{\mathbb{K}(q)} \\
& +2(1+\xi) A \int_{p}^{\alpha} d q \frac{q^{2} R(Z, \Sigma)}{\mathbb{K}(q)}\left(\frac{2}{\alpha}-\frac{2}{q}-\frac{p^{2}}{\alpha q^{2}}\right) \\
& +2(1-\xi) A \int_{0}^{p} d q \frac{q^{2} S(p, q ; Z, \Sigma)}{\mathbb{K}(q)} \\
& \times\left(\frac{1}{q} \frac{1}{p^{2}-q^{2}}-\frac{2}{\alpha p^{2}}\right)+2(1-\xi) A \\
& \times \int_{p}^{\alpha} d q \frac{q^{2} S(p, q ; Z, \Sigma)}{\mathbb{K}(q)}\left(\frac{1}{p} \frac{1}{q^{2}-p^{2}}-\frac{2}{\alpha q^{2}}\right) .
\end{aligned}
$$

The kernels are infrared singular; this arises from the propagation of a supersymmetric gauge artifact [15], namely, the lowest component of the gauge superfield; this singular behavior in the infrared is a general problem for superspace formulations of gauge field theories, but is absent in supersymmetric Feynman gauge [8]. Note that the singular terms 
as $p \rightarrow q$ are regulated on account of the vanishing of the functions $Q$ and $S$ in this same limit.

It is also evident from Eqs. (6.5) and (6.6) that it is impossible to derive from them an equivalent differential equation (not even within the bifurcation method) on account of the presence of factors of $Z(p)$ and $\Sigma(p)$ in the kernels. Furthermore, it is evident that even if the factors of $Z(p)$ and $\Sigma(p)$ could be dealt with, the same problem arises from the $\left(p^{2}-q^{2}\right)^{-1}$ terms: the integrals cannot be entirely removed by differentiation. However, this last problem is removed in the supersymmetric Feynman gauge, in which the problem of the gauge artifact discussed above is eliminated: for this reason much of our analysis will be performed in Feynman gauge, or using approximations which are computationally equivalent to this gauge.

\section{A. Wave function renormalization}

In the normal phase, where $\Sigma=0$, the integral equation for $Z$ can be integrated directly. We begin with the approximation $Z(p)=Z(q)$, which is not quite as drastic as $Z=1$, but has the same effect, namely to reduce the kernels to known functions of $p$ and $q$. Performing the integrations yields the following form for $Z$ [compare with Eq. (5.6)]:

$$
\begin{aligned}
Z(p)= & 1+\frac{2(1+\xi)}{N \pi^{2}}-\frac{8}{3} \frac{(1+\xi)}{N \pi^{2}}\left(\frac{p}{\alpha}\right)+\frac{(1+\xi)}{N \pi^{2}}\left(\frac{p}{\alpha}\right)^{2} \\
& +2 \frac{(1+\xi)}{N \pi^{2}} \ln \left(\frac{p}{\alpha}\right) \\
\simeq & a+\left(\frac{p}{\alpha}\right)^{\gamma}+f\left(\frac{p}{\alpha}\right) \\
\gamma= & 2 \frac{(1+\xi)}{N \pi^{2}}
\end{aligned}
$$

where we have used the usual renormalization group argument to resum the logarithm, and as in Eq. (5.6), $a$ is a constant of order $\mathcal{O}(1 / N)$ and the function $f$ vanishes at least as fast as $p / \alpha$ in the limit $p / \alpha \rightarrow 0$. The critical exponent $\gamma$ is found to have the usual $1 / N$ behavior. Note that the evaluation of the exponent $\gamma$ in this more reliable approach differs by a factor of 2 from the result obtained in Sec. V; this is of little concern for the exponent is not a gauge invariant object. The $N$ dependence is the crucial property, and this is the same in both computations.

We can demonstrate that this solution is stable, by now relaxing the assumption that $Z(p)=Z(q)$, and feeding the solution

$$
Z(q)=\left(\frac{q}{\alpha}\right)^{\gamma}
$$

back into the integral equation. The parts of the integral equation which vanish in Feynman gauge now give divergent contributions, in the form of hypergeometric functions evaluated at the limit of their radius of convergence, and divergent logarithms from the remaining singularities at $p$ $=q$. These singularities are again a consequence of the propagation of a supersymmetric gauge artifact; they can be successfully avoided by considering the supersymmetric Feynman gauge $\xi=1$, in which the iterated solution reads

$$
\begin{aligned}
Z(p) & =\frac{16}{3-\gamma} \frac{1}{N \pi^{2}}\left(\frac{p}{\alpha}\right)-\frac{2}{1+\gamma} \frac{1}{N \pi^{2}}\left(\frac{p}{\alpha}\right)^{2}+\left(\frac{p}{\alpha}\right)^{\gamma} \\
\gamma & =\frac{4}{N \pi^{2}} .
\end{aligned}
$$

The critical exponent is unaltered, and the coefficients of the other terms receive corrections of order $\mathcal{O}(1 / N)$ in the denominator. Hence, for large $N$ the solution is stable to iteration, at least in supersymmetric Feynman gauge.

\section{B. Self-energy}

We turn now to the self-energy, and the integral equation (6.6). As we discussed previously, it is impossible to derive an equivalent differential equation, on account of the presence of $\Sigma(p)$ and $\left(p^{2}-q^{2}\right)^{-1}$ terms in the kernels. We again study the equation in the Feynman gauge, which removes the difficulties of the singular terms. Dividing through by $Z(p)$, we obtain the following integral equation for the mass function $M(p)$ :

$$
\begin{aligned}
\left(\frac{1}{2 N \pi^{2}}\right)^{-1} M(p)= & \frac{2}{\alpha p^{2}} \int_{0}^{p} d q \frac{q^{4}}{Z(q)} \frac{M(p)-M(q)}{q^{2}+M^{2}(q)} \\
& -4 \int_{p}^{\alpha} d q \frac{q}{Z(q)} \frac{M(p)-M(q)}{q^{2}+M^{2}(q)} \\
& +\frac{4}{\alpha} \int_{p}^{\alpha} d q \frac{q^{2}}{Z(q)} \frac{M(p)-M(q)}{q^{2}+M^{2}(q)} \\
& -\frac{2 p^{2}}{\alpha} \int_{p}^{\alpha} d q \frac{1}{Z(q)} \frac{M(p)-M(q)}{q^{2}+M^{2}(q)} .
\end{aligned}
$$

To study this we cannot use the usual method of setting $M(p)=M(q)=M(0)$ everywhere and looking for selfconsistent solutions, for in this limit the kernels reduce to zero. Instead, we recall the solution of the differential equation in Sec. V, Eq. (5.9), and adopt the following ansatz for $M$ inside the kernels:

$$
M(q)=m_{0} e^{-p^{2} / \alpha^{2}} .
$$

We adopt also the solution

$$
Z(q)=\left(\frac{q}{\alpha}\right)^{\gamma}
$$

for the wave function renormalization factors which appear 
above. Unfortunately the integrations cannot be performed analytically if the exponential factor in Eq. (6.12) is present in the denominators. Hence in the denominators we set $M$ to a constant $\bar{m}$, and it is with this in mind that the scale $\bar{m}$ will be interpreted as an average mass over the range $p \in[0, \alpha]$; we will see that in the limits we wish to consider, dependence on the introduced scale drops out. We can then perform the integrations analytically with the result

$$
\begin{aligned}
\frac{M(p)}{m_{0}}= & -\frac{\gamma \pi}{4}\left(\frac{\alpha}{\bar{m}}\right)^{\gamma} e^{-p^{2} / \alpha^{2}} \csc \left(\frac{2-\gamma}{2} \pi\right)+\frac{1}{2} e^{-p^{2} / \alpha^{2}}-\frac{\gamma}{4} \Gamma\left[-\frac{\gamma}{2},\left(\frac{\bar{m}}{\alpha}\right)^{3 / 2}\right]+\frac{\gamma}{4}\left(\frac{\alpha}{\bar{m}}\right)^{\gamma} e^{\bar{m}^{2} / \alpha^{2}} \Gamma\left(\frac{2-\gamma}{2}\right) \Gamma\left(\frac{\gamma}{2}, \frac{\bar{m}^{2}}{\alpha^{2}}\right) \\
& -\frac{\gamma}{2} \frac{1}{1-\gamma}\left(\frac{p}{\alpha}\right)^{1-\gamma} e^{-p^{2} / \alpha^{2}}-\frac{1}{2} \frac{\gamma}{2-\gamma}\left(\frac{\alpha}{\bar{m}}\right)^{\gamma}\left(\sqrt{\frac{p}{\bar{m}}}{ }^{(2-\gamma) / 2}{ }_{2} F_{1}\left(1, \frac{2-\gamma}{2} ; \frac{4-\gamma}{2},-\sqrt{\frac{p}{\bar{m}}}\right)+\frac{\gamma}{2} \frac{1}{1-\gamma} e^{-p^{2} / \alpha^{2}}\right. \\
& -\frac{\gamma \pi}{4}\left(\frac{\bar{m}}{\alpha}\right) e^{1-\gamma} e^{-p^{2} / \alpha^{2}} \csc \left(\frac{1-\gamma}{2} \pi\right)+\frac{1}{2} \frac{\gamma}{1-\gamma}\left(\frac{p}{\alpha}\right)^{1-\gamma} e^{-p^{2} / \alpha^{2}}-\frac{1}{2} \frac{\gamma}{1+\gamma}\left(\frac{\bar{m}}{\alpha}\right)^{2} e^{-p^{2} / \alpha^{2}} \\
& -\frac{\gamma}{4}\left(\frac{\bar{m}}{\alpha}\right) e^{1-\gamma} \bar{m}^{2} / \alpha^{2} \Gamma\left(\frac{3-\gamma}{2}\right) \Gamma\left(\frac{\gamma-1}{2}, \frac{\bar{m}}{\alpha^{2}}\right)+\frac{1}{2} \frac{\gamma}{3-\gamma}\left(\frac{\bar{m}}{\alpha}\right)^{1-\gamma}\left(\sqrt{\frac{p}{\bar{m}}}\right) / 2 \\
& \times{ }_{2} F_{1}\left(1, \frac{3-\gamma}{2} ; \frac{5-\gamma}{2},-\sqrt{\frac{p}{\bar{m}}}\right)+\frac{\gamma}{4} \Gamma\left[\frac{1-\gamma}{2},\left(\frac{\bar{m}}{\alpha}\right)^{3 / 2}\right]+\mathcal{O}\left(\frac{p^{2}}{\alpha^{2}}\right),
\end{aligned}
$$

where

$$
\gamma=\frac{4}{N \pi^{2}}
$$

The limit $p \rightarrow 0$ is now well defined, and if we also assume $\bar{m} \ll \alpha$ and take the limit $\gamma \ll 1$ we find that

$$
\frac{M(0)}{m_{0}}=\frac{1}{1-\gamma}+\frac{\gamma}{2}\left(\frac{\pi}{2}-\frac{1}{1-\gamma}\right)+\cdots,
$$

where the dots represent the omission of terms smaller than the finite terms exhibited above. It is clear then, that to leading order in $1 / N$, the ansatz (6.12) is stable to iteration, and we have found that the solution of the differential equation in Sec. V is consistent with the full vertex and the restoration of the mass in the denominators of the kernels. We have therefore demonstrated the possibility of a finite dynamically generated mass for this theory, though we still find no evidence for a critical flavor number: we return to a discussion of this issue in Sec. IX.

\section{Concluding remarks}

In this section we have presented a computation of the wave function renormalization and self-energy with a threepoint vertex which is consistent with the U(1) Ward identity. While we have not been able to use the elegant methods of Sec. V, we have been able to perform a direct computation, and within the approximations made we have found that the wave function renormalization exhibits the expected critical behavior [16-18] and we have exhibited a finite solution to the gap equation for the mass function

$$
\begin{gathered}
Z(p) \simeq\left(\frac{p}{\alpha}\right)^{\gamma}, \quad \gamma=2 \frac{(1+\xi)}{N \pi^{2}}, \\
M(p)=m_{0} e^{-p^{2} / \alpha^{2}} .
\end{gathered}
$$

These results are qualitatively the same as those derived using the simple approximations of Sec. V, justifying the use of those approximations in determining qualitatively the gauge dependence in this model.

Since we have found that a mass can be dynamically generated in this model without breaking supersymmetry, it is natural to ask whether it is possible to determine whether the massive or massless (for $m_{0}$ could still vanish) solution is favored. In nonsupersymmetric theories this question can be addressed by appealing to the effective potential (which upon using a suitable variational principle leads to the same nonperturbative physics for $\mathrm{QED}_{3}$ as do the DysonSchwinger equations [24]), but in supersymmetric models this potential vanishes. Based on experience with supersymmetric nonlinear sigma models [19] it was conjectured [1] that the question could be settled by appealing to the effective action, and to the Ward identities arising from the supersymmetry. The same approach was taken in Ref. [21] for a [(2+1)-dimensional] supersymmetric model with a $C P^{1}$ constraint. The simplest Ward identity arising from supersymmetry relates the two-point correlation functions of the physical degrees of freedom in the matter superfield (in the presence of a mass $m$ for the multiplet): 


$$
\left\langle\psi_{\alpha}(x) \psi_{\beta}(y)\right\rangle_{0}=\left(\partial_{\alpha \beta}+m C_{\alpha \beta}\right)\langle\phi(x) \phi(y)\rangle_{0} .
$$

Crucially, in the models of Refs. [19-21], where there are further similar Ward identities relating correlation functions of fields arising from the implementation of the constraints, it was found that these Ward identities were only satisfied when the mass for the matter multiplet was nonvanishing. In the present model, and in the $\mathcal{N}=2$ model of Ref. [1], there are no such constraints, and so the Ward identities place no further requirements on the generated mass; of course, the superfield formalism we have adopted means that the Ward identity (6.16) is satisfied automatically. This leaves open the question of whether the vacuum selects the massless or massive solution in supersymmetric U(1) gauge field theory.

\section{EXTENDED SUPERSYMMETRIC ACTION}

The $\mathcal{N}=2$ extended rigid superspace is constructed in a way slightly different to the $\mathcal{N}=1$ model: it can be obtained by dimensional reduction from $\mathcal{N}=1$ supersymmetry in four dimensions. The compactification is realised by considering all the fields in the model to be independent of one spatial dimension and then integrating out this variable. The rigid superspace of four dimensional $\mathcal{N}=1$ supersymmetry is parameterized by the usual four space-time coordinates and four Grassmann-odd coordinates arranged in two spinors $\theta^{\alpha}$ and $\bar{\theta}^{\dot{\alpha}}$, where $\alpha \in\{1,2\}$ and $\dot{\alpha} \in\{\dot{1}, \dot{2}\}$ [25], which we again denote collectively by $z$. This furnishes the superspace with two spinorial derivatives $D_{\alpha}$ and $\bar{D}_{\alpha}$, covariant under supersymmetry transformations, which leads to a crucial difference compared to the three-dimensional model, in that the matter superfields now come in two types: chiral $(\Phi)$ and antichiral $\left(\Phi^{\dagger}\right)$, which respectively obey the constraints

$$
\bar{D}_{\alpha} \Phi=0, \quad D_{\alpha} \Phi^{\dagger}=0 .
$$

We collect some basic results for four dimensional superspace in Appendix B. Consider chiral and antichiral superfields which transform under local U(1) transformations in the following way:

$$
\begin{aligned}
& \Phi_{ \pm}(z) \rightarrow \Phi_{ \pm}^{\prime}\left(z^{\prime}\right)=e^{ \pm i e \Lambda(z)} \Phi_{ \pm}(z), \\
& \Phi_{ \pm}^{\dagger}(z) \rightarrow \Phi_{ \pm}^{\dagger^{\prime}}\left(z^{\prime}\right)=\Phi_{ \pm}^{\dagger}(z) e^{\overline{\mp i e} \bar{\Lambda}(z)} .
\end{aligned}
$$

The functions $\Lambda$ and $\bar{\Lambda}$ must respectively be chiral and antichiral, to preserve the chiral and antichiral nature of the matter superfields. Note that $e$ is a dimensionless gauge coupling: the dimensionful parameter which will again lead to a dynamically generated scale will be the size of the compactified dimension. The gauge field is now a real scalar superfield, and transforms under infinitesimal U(1) transformations in the following way:

$$
V(z)=V^{\dagger}(z) \rightarrow V^{\prime}\left(z^{\prime}\right)=V(z)+\frac{i}{2}(\bar{\Lambda}-\Lambda) .
$$

We now wish to consider a model with $\mathcal{N}=1$ supersymmetry, local U(1) gauge invariance and $N$ matter flavors; again the action functional comprises three parts, a locally $\mathrm{U}(1)$ invariant kinetic term for the matter superfields, a gauge invariant classical field strength for the gauge superfield, a (Lorentz) gauge fixing term; for convenience we will also add a bare mass term for the matter superfields:

$$
\begin{gathered}
S=S_{g}^{\text {class }}+S_{g}^{\mathrm{GF}}+S_{m}^{\mathrm{k}}+S_{m}^{\mathrm{m}}, \\
S_{g}^{\mathrm{class}}=\int d^{4} x d^{4} \theta V\left[\frac{1}{8} D^{\alpha} \bar{D}^{2} D_{\alpha}\right] V \\
S_{g}^{\mathrm{GF}}=\int d^{4} x d^{4} \theta \frac{1}{8} V\left[-\frac{1}{8 \xi} D^{2} \bar{D}^{2}\right] V, \\
S_{m}^{\mathrm{k}}=\int d^{4} x d^{4} \theta\left[\Phi_{+}^{\dagger} e^{2 e V} \Phi_{+}+\Phi_{-}^{\dagger} e^{-2 e V} \Phi_{-}\right], \\
S_{m}^{\mathrm{m}}=\int d^{4} x d^{2} \theta \mu_{0} \Phi_{+} \Phi_{-}+\int d^{4} x d^{2} \bar{\theta} \mu_{0} \Phi_{+}^{\dagger} \Phi_{-}^{\dagger} .
\end{gathered}
$$

Again we have included an implicit sum over $N$ flavors in the matter parts. There are a number of important differences between this action and the one we considered in the first part of this paper. First, there are now twice as many matter superfields: an antichiral and chiral superfield for each charge under U(1). As we shall see this changes considerably the structure of the propagators; in particular, note that in the absence of a bare mass $\mu_{0}$ the noninteracting correlation function $\left\langle\Phi_{+} \Phi_{-}\right\rangle_{0}$ vanishes, and the Dyson-Schwinger equations for the self-energy and wave function renormalization are decoupled to an extent. Furthermore, it is evident that the implementation of the interaction with the gauge field is completely different: there are now an infinite number of vertices with increasing numbers of gauge superfields. In the Wess-Zumino gauge (which projects out the physical components of the gauge superfield, but which thereby breaks supersymmetry explicitly) the sequence of interaction vertices terminates at the four point vertex, giving it the same content as the model considered in the first part of this paper. In order to make the model tractable, we will truncate the Dyson-Schwinger equations at the level of the four-point vertex, effectively setting higher-point vertices to zero [8]. This situation is certainly not ideal [9], but we find that the graph in the Dyson-Schwinger equation arising from the four-point vertex contributes trivially to the wave function renormalization, and we therefore believe that this truncation will not affect the infrared physics. The dressed propagators for the model are as follows:

$$
\begin{aligned}
& \Delta^{\Phi_{+} \Phi_{-}(p ; 12)}=\frac{i \Sigma(p)}{Z^{2}(p) p^{2}+\Sigma^{2}(p)} \frac{\bar{D}^{2}}{4} \delta^{4}(12), \\
& \Delta_{ \pm}^{\Phi_{ \pm}^{\dagger} \Phi_{ \pm}(p ; 12)}=\frac{i Z(p)}{Z^{2}(p) p^{2}+\Sigma^{2}(p)} \frac{D^{2} \bar{D}^{2}}{16} \delta^{4}(12),
\end{aligned}
$$




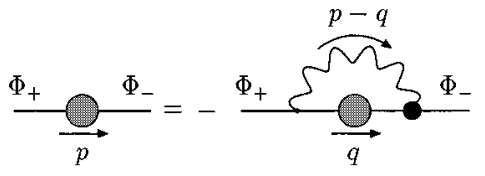

FIG. 2. Schematic form of the Dyson-Schwinger equation for the self-energy function. Solid lines represent matter superfields, and the wavy line represents the gauge superfield; blobs indicate full nonperturbative quantities.

we have again used an abbreviated superspace notation where, for example,

$$
\begin{aligned}
\delta^{4}(12) & \equiv \delta^{4}\left(\theta_{1}-\theta_{2}\right)=\delta^{2}\left(\theta_{1}-\theta_{2}\right) \delta^{2}\left(\bar{\theta}_{1}-\bar{\theta}_{2}\right) \\
& \equiv \delta^{2}(12) \delta^{2}(\overline{1} \overline{2}) .
\end{aligned}
$$

The undressed gauge superfield propagator reads [25]

$$
\Delta^{V V}(p ; 12)=\frac{1}{2 p^{2}} e^{\theta_{1} p \bar{\theta}_{2}-\theta_{2} p \theta_{1}}\left\{\frac{4}{p^{2}}(1-\xi)-(1+\xi) \delta^{4}(12)\right\} ;
$$

later we will dress this propagator with a vacuum polarization factor as before. For simplicity we will restrict our attention to the simple vertex approximation considered in the approach of Sec. V; this is in fact consistent with the U(1) Ward identity for this model [8], at least in the limit of vanishing transferred momentum $p$.

\section{THE DYSON-SCHWINGER EQUATIONS}

We can rewrite the matter superfields in terms of unconstrained scalar superfields, which makes the evaluation of the superspace parts of the Dyson-Schwinger equations simpler:

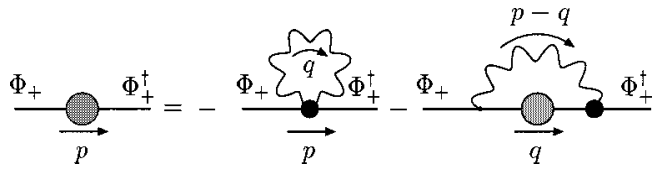

FIG. 3. Schematic form of the Dyson-Schwinger equation for the wave-function renormalization. Solid lines represent matter superfields and wavy lines represent gauge superfields; blobs indicate full nonperturbative quantities.

$$
\begin{aligned}
& \Phi_{ \pm}(p, \theta, \bar{\theta})=e^{-\theta p \bar{\theta}} \Psi_{ \pm}(p, \theta), \\
& \Phi_{ \pm}^{\dagger}(p, \theta, \bar{\theta})=e^{\theta p \bar{\theta}} \Psi_{ \pm}^{\dagger}(p, \bar{\theta}) .
\end{aligned}
$$

The Dyson-Schwinger equations for the current model are shown schematically in Figs. 2 and 3. The graphs on the left hand side are a convenient abbreviation for the difference between the inverse full propagator and inverse bare propagator.

The full self-energy function on the left hand side of Fig. 2 is written as follows:

$$
\begin{aligned}
\int & d^{2} \theta_{1} d^{2} \theta_{2} \Phi_{+}\left(-p, \theta_{2}\right) \Sigma(p) \frac{\bar{D}^{2}}{4} \delta^{4}(12), \Phi_{-}\left(p, \theta_{1}\right) \\
= & \int d^{2} \theta_{1} d^{2} \theta_{2} e^{\theta_{2} p \bar{\theta}_{2}} \Psi_{+}\left(-p, \theta_{2}\right) \Sigma(p) \\
& \times \delta^{2}(12) e^{-\theta_{1} p\left(\bar{\theta}_{1}-\bar{\theta}_{2}\right)} e^{-\theta_{1} p \bar{\theta}_{1}} \Psi_{-}\left(p, \theta_{1}\right) \\
= & \int d^{2} \theta \Psi_{+}(-p, \theta) \Sigma(p) \Psi_{-}(p, \theta) .
\end{aligned}
$$

The graph on the right of Fig. 2 is given by the following $(P \doteq p-q)$ :

$$
\begin{aligned}
\int & \frac{d^{4} q}{(2 \pi)^{4}} d^{4} \theta d^{4} \bar{\theta} \Phi_{+}\left(-p, \theta_{2}\right)\left[\left(-4 e^{2} G_{3}\right) \Delta^{\Phi_{+}^{\dagger}} \Phi_{-}^{\dagger}(q ; 12) \Delta^{V V}(P ; 12)\right], \Phi_{-}\left(p, \theta_{1}\right) \\
= & -\int \frac{d^{4} q}{(2 \pi)^{4}} d^{4} \theta d^{4} \bar{\theta} 4 e^{2} G_{3} \Phi_{+}\left(-p, \theta_{2}\right) \\
& \times \frac{i \Sigma(q)}{Z^{2}(q) q^{2}+\Sigma^{2}(q)} \frac{D^{2}}{4} \delta^{4}(12) \frac{1}{2 P^{2}} e^{\theta_{1} p \bar{\theta}_{2}-\theta_{2} p \bar{\theta}_{1}}\left\{\frac{4}{P^{2}}(1-\xi)-(1+\xi) \delta^{4}(12)\right\} \Phi_{-}\left(p, \theta_{1}\right) .
\end{aligned}
$$

Expanding the exponentials and performing half of the superspace integrations using the identities in Appendix B leaves

$$
i \int \frac{d^{4} q}{(2 \pi)^{4}} 4 e^{2} \Gamma_{3} \frac{\Sigma(q)}{Z^{2}(q) q^{2}+\Sigma^{2}(q)} \frac{2 p^{2}}{P^{4}}(1-\xi) \int d^{2} \theta \Psi_{+}(-p, \theta) \Psi_{-}(p, \theta) .
$$

Note that in this model the superspace integrations project out the $(1-\xi)$ component of this graph. This is the basis of the gauge dependence argument [8] for the nongeneration of dynamical mass in this theory.

Turning to Fig. 3, the graph on the left for the full wave function renormalization is written as follows: 


$$
\begin{aligned}
& \int d^{2} \theta_{1} d^{2} \bar{\theta}_{2} \Phi_{+}\left(-p, \theta_{2}\right)[Z(p)-1] \frac{\bar{D}^{2} D^{2}}{16} \delta^{4}(12) \Phi_{+}^{\dagger}\left(p, \theta_{1}\right) \\
& =\int d^{2} \theta_{1} d^{2} \bar{\theta}_{2} e^{\theta_{2} p \bar{\theta}_{2}} \Psi_{+}\left(-p, \theta_{2}\right)[Z(p)-1] e^{-\theta_{1} p \bar{\theta}_{1}-\theta_{2} p \bar{\theta}_{2}+2 \theta_{1} p \bar{\theta}_{2}} e^{\theta_{1} p \bar{\theta}_{1}} \Psi_{+}^{\dagger}\left(p, \bar{\theta}_{1}\right) \\
& =\int d^{2} \theta_{1} d^{2} \bar{\theta}_{2} \Psi_{+}\left(-p, \theta_{2}\right)[Z(p)-1] e^{2 \theta_{1} p \bar{\theta}_{2}} \Psi_{+}^{\dagger}\left(p, \bar{\theta}_{1}\right)=\int d^{4} \theta[Z(p)-1] \Phi_{+}(-p, \theta, \bar{\theta}) \Phi_{+}^{\dagger}(p, \theta, \bar{\theta}) .
\end{aligned}
$$

The superspace integrations can be performed in the same way as for the self-energy to write the graphs on the right of Fig. 3. The "seagull" graph is computed to be a trivial $p$ independent contribution, as in the model of the first part of this paper (and which again vanishes in supersymmetric Feynman gauge):

$$
\int \frac{d^{4} q}{(2 \pi)^{4}} d^{4} \theta \frac{1}{2} 4 e^{2} G_{4} \Phi_{+}(-p, \theta, \bar{\theta}) \Delta^{V V}(q) \Phi_{+}^{\dagger}(p, \theta, \bar{\theta})=\int \frac{d^{4} q}{(2 \pi)^{4}} 2 e^{2} \Gamma_{4} \frac{2}{P^{4}}(1-\xi) \int d^{4} \theta \Phi_{+}^{\dagger}(p, \theta, \bar{\theta}) \Phi_{+}(-p, \theta, \bar{\theta}) .
$$

The last graph on the right of Fig. 3 is as follows:

$$
\begin{aligned}
& \int \frac{d^{4} q}{(2 \pi)^{4}} d^{4} \theta_{1} d^{4} \theta_{2} \Phi_{+}\left(-p, \theta_{2}\right) 4 e^{2} G_{3} \Delta^{\Phi_{+}^{\dagger} \Phi_{+}(q ; 12) \Delta^{V V}(P ; 12) \Phi_{+}^{\dagger}\left(p, \theta_{1}\right)} \\
& =\int \frac{d^{4} q}{(2 \pi)^{4}} 4 e^{2} G_{3} \frac{i Z(q)}{Z^{2}(q) q^{2}+\Sigma^{2}(q)}\left[\frac{(p+q)^{2}}{2 P^{4}}(1-\xi)-(1+\xi)\right] \int d^{4} \theta \Phi_{+}(-p, \theta, \bar{\theta}) \Phi_{+}^{\dagger}\left(p, \theta_{1}\right) .
\end{aligned}
$$

Collecting the results above, the Dyson-Schwinger equations of Figs. 2 and 3 then yield the following coupled integral equations for the self energy and wave function renormalization:

$$
\begin{aligned}
\Sigma(p)= & -i 4 e^{2} p^{2}(1-\xi) \\
& \times \int \frac{d^{4} q}{(2 \pi)^{4}} G_{3} \frac{\Sigma(q)}{Z^{2}(q) q^{2}+\Sigma^{2}(q)} \frac{2}{(p-q)^{4}},
\end{aligned}
$$

$$
\begin{aligned}
Z(p)= & 1-i 4 e^{2}(1+\xi) \\
& \times \int \frac{d^{4} q}{(2 \pi)^{4}} G_{3} \frac{Z(q)}{Z^{2}(q) q^{2}+\Sigma^{2}(q)} \frac{1}{2(p-q)^{2}} \\
& +i 4 e^{2}(1-\xi) \int \frac{d^{4} q}{(2 \pi)^{4}} G_{3} \frac{Z(q)}{Z^{2}(q) q^{2}+\Sigma^{2}(q)} \frac{(p+q)^{2}}{(p-q)^{4}} \\
& +4 e^{2}(1-\xi) \int \frac{d^{4} q}{(2 \pi)^{4}} G_{4} \frac{1}{q^{4}} .
\end{aligned}
$$

To study the infrared physics of $\mathcal{N}=2$ supersymmetric $\mathrm{U}(1)$ gauge field theory in three dimensions, we will simply consider all the fields above to be independent of one coordinate, and then integrate it out: this will introduce a natural scale $\rho$, the size of the compactified dimension, and we will then be able to compare directly with the model of the first part of this paper. We compactify one dimension as follows:

$$
\int d q_{3}=\frac{1}{\rho}
$$

We can now incorporate the effects of massless matter loops into the gauge field propagator, by dressing it with the appropriate vacuum polarization factor:

$$
\Delta^{V V}(p ; 12) \rightarrow \frac{\Delta^{V V}(p ; 12)}{1+\bar{\alpha} /|p|}
$$

where we have introduced the ultraviolet scale

$$
\bar{\alpha} \doteq \frac{e^{2} N}{\rho}
$$

in analogy with the treatment of normal $\mathrm{QED}_{3}$ and the $\mathcal{N}$ $=1$ model considered in the first part of this paper. The combination $e^{2} / \rho$ relates the dimensionless coupling $e$ of the original model to the dimensionful coupling in the dimensionally reduced theory. We can carry out the angular part of the momentum integration easily in three dimensions, with result 


$$
\begin{aligned}
& \Sigma(p)=\frac{\bar{\alpha}}{2 N \pi^{3}}(1-\xi) \int d q G_{3} \frac{\Sigma(q)}{Z^{2}(q) q^{2}+\Sigma^{2}(q)} 2 q p\left\{\frac{1}{\bar{\alpha}}\left(\frac{1}{|p-q|}-\frac{1}{p+q}\right)+\frac{1}{\bar{\alpha}^{2}} \ln \left[\frac{p+q+\bar{\alpha}}{|p-q|+\bar{\alpha}}\right]-\frac{1}{\bar{\alpha}^{2}} \ln \left[\frac{p+q}{|p-q|}\right]\right\}, \\
& Z(p)=+\frac{\bar{\alpha}}{2 N \pi^{3}}(1+\xi) \int d q G_{3} \frac{Z(q)}{Z^{2}(q) q^{2}+\Sigma^{2}(q)} \frac{q}{2 p} \ln \left[\frac{p+q+\bar{\alpha}}{|p-q|+\bar{\alpha}}\right] \\
&-\frac{\bar{\alpha}}{2 N \pi^{3}}(1-\xi) \int d q G_{3} \frac{Z(q)}{Z^{2}(q) q^{2}+\Sigma^{2}(q)} \frac{q}{2 p}\left\{\frac{2\left(p^{2}+q^{2}\right)}{\bar{\alpha}}\left(\frac{1}{|p-q|}-\frac{1}{p+q}\right)\right. \\
&\left.-\frac{2\left(p^{2}+q^{2}\right)}{\bar{\alpha}^{2}} \ln \left[\frac{p+q}{|p-q|}\right]+\frac{2\left(p^{2}+q^{2}\right)-\bar{\alpha}^{2}}{\bar{\alpha}^{2}} \ln \left[\frac{p+q+\bar{\alpha}}{|p-q|+\bar{\alpha}}\right]\right\}-\frac{i \bar{\alpha}}{2 N \pi^{3}}(1-\xi) \\
& \times \int d q \frac{q}{p} G_{4}\left\{\frac{1}{\bar{\alpha}}\left(\frac{1}{|p-q|}-\frac{1}{p+q}\right)-\frac{1}{\bar{\alpha}^{2}} \ln \left[\frac{p+q}{|p-q|}\right]+\frac{1}{\bar{\alpha}^{2}} \ln \left[\frac{p+q+\bar{\alpha}}{|p-q|+\bar{\alpha}}\right]\right\} .
\end{aligned}
$$

To study these equations it is once again necessary to expand the logarithms arising from the angular integrations, in the limit $p, q \ll \bar{\alpha}$, which leaves

$$
\begin{aligned}
\Sigma(p)=\frac{\bar{\alpha}}{N \pi^{3}}(1-\xi) \int d q \mathcal{F}(q)\left[\frac{2 q^{2} p}{p^{2}-q^{2}} \theta(p-q)+\frac{2 p^{2} q}{q^{2}-p^{2}} \theta(q-p)\right], \\
Z(p)=1+\frac{\bar{\alpha}}{2 N \pi^{3}}(1+\xi) \int d q \mathcal{G}(q)\left[\frac{q^{2}}{\bar{\alpha} p} \theta(p-q)+\frac{q}{\bar{\alpha}} \theta(q-p)\right] \\
-\frac{\bar{\alpha}}{2 N \pi^{3}}(1-\xi) \int d q \mathcal{G}(q)\left[\left(\frac{p^{2}+3 q^{2}}{p^{2}-q^{2}}\right) \frac{q^{2}}{\bar{\alpha} p} \theta(p-q)+\left(\frac{3 p^{2}+q^{2}}{q^{2}-p^{2}}\right) \frac{q}{\bar{\alpha}} \theta(q-p)\right] \\
-\frac{i \bar{\alpha}}{2 N \pi^{3}}(1-\xi) \int d q \Gamma_{4}\left[\frac{2 q^{2}}{\bar{\alpha} p} \frac{1}{p^{2}-q^{2}} \theta(p-q)+\frac{2 q}{\bar{\alpha}} \frac{1}{q^{2}-p^{2}} \theta(q-p)\right],
\end{aligned}
$$

where we have introduced the convenient abbreviated notation:

$$
\begin{aligned}
& \mathcal{F}(q)=G_{3} \frac{\Sigma(q)}{Z^{2}(q) q^{2}+\Sigma^{2}(q)}, \\
& \mathcal{G}(q)=G_{3} \frac{Z(q)}{Z^{2}(q) q^{2}+\Sigma^{2}(q)} .
\end{aligned}
$$

\section{A. Wave function renormalization}

In the normal phase (or equivalently, within the bifurcation method) where $\Sigma=0$, the integral equation for the wave function renormalization can be integrated directly upon using the simple approximation $G_{3}=Z(q), G_{4}=1[8]$

$$
\begin{aligned}
Z(p)= & 1+\frac{\bar{\alpha}}{2 N \pi^{3}}(1+\xi)\left[\frac{1}{\bar{\alpha}}-\frac{1}{\bar{\alpha}} \ln \left(\frac{p}{\bar{\alpha}}\right)\right]-\frac{\bar{\alpha}}{2 N \pi^{3}}(1-\xi)\left[-\frac{3}{\bar{\alpha}}+\frac{4}{\bar{\alpha}} \tanh ^{-1}\left(1-\frac{\varepsilon}{p}\right)\right] \\
& -\frac{\bar{\alpha}}{2 N \pi^{3}}(1-\xi)\left[\frac{3}{\bar{\alpha}} \ln \left(\frac{p}{\bar{\alpha}}\right)+\frac{2}{\bar{\alpha}} \ln \left(\frac{\bar{\alpha}^{2}-p^{2}}{2 p \varepsilon}\right)\right] \\
& -\frac{i \bar{\alpha}}{2 N \pi^{3}}(1-\xi)\left[-\frac{2}{\bar{\alpha}}+\frac{2}{\bar{\alpha}} \tanh ^{-1}\left(1-\frac{\varepsilon}{p}\right)+\frac{1}{\bar{\alpha}} \ln \left(\frac{\bar{\alpha}^{2}-p^{2}}{2 p \varepsilon}\right)\right]
\end{aligned}
$$


where the limit $\varepsilon \rightarrow 0^{+}$is implied. Again, outside the supersymmetric Feynmann gauge, there are many infrared divergences which result from the propagation of a gauge artifact in the gauge superfield. However, ignoring these divergences as spurious, we can see that the solution above is consistent with the expected critical behavior, with an exponent of or$\operatorname{der} \mathcal{O}(1 / N)$ :

$$
Z(p) \simeq\left(\frac{p}{\bar{\alpha}}\right)^{\bar{\gamma}}, \quad \bar{\gamma} \simeq \frac{1-2 \xi}{N \pi^{3}}
$$

While it is not obvious that this form for the wave function renormalization is completely reliable, what is clear is that there is no evidence for an overall factor of $(1-\xi)$ in the wave function renormalization, a fact which will be crucial for the discussion of the self-energy which follows.

\section{B. Self-energy}

It was argued in Ref. [8] that the overall factor of (1 $-\xi$ ) in the first of Eqs. (8.13) and its four-dimensional ancestor (8.8) for the self-energy means that the self-energy vanishes in Feynman gauge, and since the full gauge dependence was carried through, this indicates that the self-energy vanishes in all gauges; this was conjectured to be the result of a four-dimensional (nonperturbative) nonrenormalization theorem [8]. This argument has been criticized $[9,10]$ on the grounds that the self-energy function is not a gauge invariant function, nor are the functions appearing in the kernel; in particular, the gauge dependence of the functions appearing in the kernel could in principle conspire to cancel the overall factor of $(1-\xi)$ and leave a gauge independent integral equation, which could have finite solutions. To answer this question it is convenient to divide the integral equation for $\Sigma$ through by $Z(p)$ to obtain

$$
\begin{aligned}
M(p)= & \frac{\bar{\alpha}}{N \pi^{3}}(1-\xi) \int d q \frac{1}{Z(p)} \frac{M(q)}{q^{2}+M^{2}(q)} \\
& \times\left[\frac{2 q^{2} p}{p^{2}-q^{2}} \theta(p-q)+\frac{2 p^{2} q}{q^{2}-p^{2}} \theta(q-p)\right] .
\end{aligned}
$$

In the limit $p \rightarrow 0$ the function $M(p)$ is gauge invariant; since we have shown that there can be no factor of $(1-\xi)$ from the wave function renormalization factor in Eq. (8.17), it seems that the function $M(p)$, even if the result of integrating the kernel were finite, would retain the overall factor of $(1-\xi)$, and therefore the only self-consistent solution would be that the mass vanished. We have not probed the structure of the vertex $G_{3}$ coming from the U(1) Ward identity [8], but this will only relate $G_{3}$ to combinations of $\Sigma$ and $Z$ : it will not lead to the gauge dependence required to cancel the overall factor of $(1-\xi)$. In order to make this argument, we depend on the approximate form of the wave function renormalization (8.16), and hence on the scale $\bar{\alpha}$; therefore our argument does not extend back to the four-dimensional model where the scale $\bar{\alpha}$ is absent, and the coupling remains dimensionless.

A related point of interest is that, contrasting the $\mathcal{N}=1$ case, there is no part of the integral equation for $M(p)$ which is not troubled by the infrared divergences generated by the gauge artifact; these divergences are known to disappear in supersymmetric Feynman gauge. The structure of the divergent terms make it impossible to construct an equivalent differential equation from the integral equation above.

\section{Concluding remarks}

In this part of the paper we have attempted to probe carefully the gauge dependence of the $\mathcal{N}=2$ model. We have chosen a simplified three vertex and we have truncated the infinite series of higher-point vertices at four. Within these approximations, we have been able to compute the wave function renormalization, and have shown that although the computation is plagued with (expected) infrared divergences, we find no evidence for the overall gauge dependence which would be necessary to cancel the factor of $(1-\xi)$ in Eq. (8.17) and evade the gauge dependence argument of Ref. [8]. We have followed this argument through for the (compactified) three-dimensional model, where the scale $\bar{\alpha}$ is available; in particular, the renormalization group resummed form for the wave function renormalization requires the existence of the scale $\bar{\alpha}$. While the properties of the four-dimensional model are communicated to the three-dimensional extended model through the compactification, it is not clear that the results presented here are applicable also for the fourdimensional model where the scale $\bar{\alpha}$ is necessarily absent; whence the arguments of Refs. $[9,10]$ on evading the nonrenormalization theorem in the four-dimensional model could still hold. Our results here are in agreement with a numerical evaluation of the effective potential for the threedimensional model in component formalism [11].

\section{DISCUSSION AND CONCLUSION}

\section{A. The $\mathcal{N}=1$ model}

The superfield formalism we have adopted has the advantage of keeping supersymmetry manifest; this has allowed us to concentrate on two aspects of the infrared physics which have troubled components computations in both the supersymmetric and nonsupersymmetric versions of this model, namely gauge invariance and the full vertex. In particular, the superfield formalism gave us extra power to deal with the $\mathrm{U}(1)$ Ward identity for the full vertex. The disadvantages of the superfield approach are apparent in the spurious singular terms which appear as a result of propagating extra degrees of freedom in the gauge superfield. This problem can be avoided in supersymmetric Feynman gauge, and it is this which means we have had to give separate treatments to probe the gauge dependence and the issue of the full vertex.

The first treatment employed some of the simplest approximations: a near trivial vertex [which satisfies the U(1) Ward identity only in the limit of vanishing transferred mo- 
mentum, but which allows for a nontrivial wave function renormalization] and neglecting the mass in the denominators of the kernels. This program of approximations is drastic, but computationally extremely convenient: enough to allow us to probe the gauge dependence of the mass function. This vertex choice and the bifurcation method have been used with some success in the study of nonsupersymmetric $\mathrm{QED}_{3}$, and is sufficient to demonstrate the existence of a dynamically generated mass [3] (at least in Landau gauge). In the model studied here, the method returns the expected critical behavior in the wave function renormalization, and exhibits a gauge independent solution for the dynamically generated mass. The critical behavior we find contrasts the trivial wave function renormalization of Ref. [12], and is qualitatively the same as that of nonsupersymmetric $\mathrm{QED}_{3}$, where mass is also dynamically generated. Our second treatment employs the biggest advantage of the superfield formalism, that of making the U(1) Ward identity tractable. Unfortunately, the problem of infrared divergences in the superspace approach restrict us to working in supersymmetric Feynman gauge for this computation. However, we find again the expected critical behavior in the wave function renormalization and again exhibit the possibility of a finite dynamically generated mass; in particular, we find that the solution obtained in the first simplified approach is consistent with the full vertex, and a nonvanishing mass function in the denominators. It is possible to criticize the approaches taken in both treatments we have presented, but since they tackle different issues with the same result, we argue that taken together they provide strong evidence for nontrivial infrared physics in $\mathcal{N}=1$ supersymmetric $\mathrm{U}(1)$ gauge theory.

The question of whether or not the finite mass solution is actually selected has been addressed: contrary to the suggestion in Ref. [1] and the situations in the constrained models of Refs. [19-21], we find that no extra information can be obtained by appealing to the supersymmetry Ward identities. Unfortunately this leaves us with no way to determine whether the finite mass solution is actually selected by the vacuum of this theory.

We have found no evidence in this model for a critical flavor number, above which no mass generation occurs. This is in contrast to the nonsupersymmetric case $[2,4]$. As has been noted already, the differential equation (5.8) has finite solutions when the mass function is gauge invariant for all $p$ (in the range permitted by our approximations: the infrared), but no solutions which are gauge invariant only in the limit of vanishing $p$ and gauge dependent elsewhere. This is to be contrasted with the nonsupersymmetric case, where solutions of the latter type do appear to exist. The integral equation for the mass function in that case [in general gauge, and with the simplified vertex choice of $\left.G_{3}=Z(q)\right]$ is as follows:

$$
\begin{aligned}
M(p)= & \frac{8}{\pi^{2} N}\left[\int_{0}^{p} d q\left(\frac{1}{p}+\frac{\xi \alpha}{2 p^{2}}\right) \frac{q^{2} M(q)}{q^{2}+M^{2}(q)}\right. \\
& \left.+\int_{p}^{\alpha} d q\left(q+\frac{\xi \alpha}{2}\right) \frac{M(q)}{q^{2}+M^{2}(q)}\right] .
\end{aligned}
$$

The conventional method for the nonsupersymmetric case is to consider Landau gauge $(\xi=0)$ and construct an equivalent differential equation [4]

$$
\frac{\partial}{\partial p}\left(p^{2} \frac{\partial M}{\partial p}\right)=-\frac{8}{\pi^{2} N} \frac{p^{2} M}{p^{2}+M^{2}} .
$$

The solution of this differential equation takes the form of a hypergeometric function; crucially this leads to a constraint on $N$ such that above a certain critical flavor number, no mass generation occurs [4]. Proceeding as we have done for the supersymmetric model, one has to make three differentiations with respect to $p$ to remove the integrations, and the linearized (neglecting the mass in the denominators) result is as follows (in dimensionless variables rescaled by $\alpha$ ):

$$
\begin{gathered}
p^{3} \dot{m}^{\prime \prime \prime}+6 p^{2} \dot{m}^{\prime \prime}+\left[6 p+\frac{8}{N \pi^{2}}(p+\xi)\right] \dot{m}^{\prime} \\
+\frac{16}{N \pi^{2}} \dot{m}+\frac{8}{N \pi^{2}} m^{\prime}=0 .
\end{gathered}
$$

Demanding as before that $\dot{m}=0$ for all $p$, we find a constant solution for $m$; substituting a gauge dependent power series indicates that there is also the possibility of gauge independent constant as $p \rightarrow 0$ but with a gauge dependent function of $p$ away from this limit. This latter type of solution does not exist in the supersymmetric model. We see then that the gauge dependence of the two models is rather subtly different, and the fact that we find no critical flavor number may be related to this. In support of this view it is instructive to attempt to construct a differential equation similar to Eq. (9.2) for the $\mathcal{N}=1$ supersymmetric model, which might then show some evidence for a critical flavor number. To this end we return to the second of integral equations (5.3) for the self-energy, and again perform differentiations with respect to $p$, but retaining the mass as a constant in the denominators [4]:

$$
\frac{\partial}{\partial p}\left(p^{2} \frac{\partial M(p)}{\partial p}\right)=\frac{2(1-4 \xi)}{N \pi^{2}} \frac{p^{2} M(p)}{p^{2}+M^{2}} .
$$

By analogy with the analysis of Ref. [4] the "critical flavor number" derived from the equation above would be

$$
N_{c}=\frac{8(1-4 \xi)}{\pi^{2}},
$$

which on account of its explicit gauge dependence has no physical meaning.

An interesting comparison can be made with the model of Ref. [26], where dynamical mass generation in an $N$-flavor effective theory of fermions in three dimensions is studied using the renormalization group and the $\epsilon$ expansion. The effective theory has for its degrees of freedom explicitly gauge independent (composite operator) fields, and so the treatment is necessarily gauge independent; in this approach also there is no critical flavor number. We also found no 
critical flavor number when we included the effects of the full vertex (and the nontrivial wave function renormalization): this is reminiscent of results in nonsupersymmetric $\mathrm{QED}_{3}$ [27] where it has been argued that the critical flavor number of Ref. [2] was an artifact of including only a trivial wave function renormalization and simplified vertex. In both our simple computation and the full computation, we have included the effects of nontrivial wave function renormalization.

\section{B. The $\mathcal{N}=2$ model}

In contrast to the $\mathcal{N}=1$ model, in extended supersymmetric electrodynamics, we find strong evidence for the nonexistence of a dynamically generated mass, based on a refinement of the gauge dependence argument of Ref. [8]. We have used the same truncation in the Dyson-Schwinger equations (in keeping only three- and four-point vertices) as used in the four-dimensional model. This truncation is consistent with restriction to the Wess-Zumino gauge, which respects neither supersymmetry nor gauge invariance. Within this approximation we have chosen a simplified vertex (but one which is consistent with a nontrivial wave function renormalization) and we have then computed the wave function renormalization. We found no evidence for the appearance of an overall factor of $(1-\xi)$ which, if present, could have evaded $[9,10]$ the gauge dependence argument of Ref. [8]. Instead we found that the wave function renormalization seems to have the same sort of critical behavior as in the nonsupersymmetric and $\mathcal{N}=1$ cases, though this is by no means certain on account of the presence of infrared divergences (again from an artifact in the gauge superfield). Our result here is in line with Ref. [11], in which a numerical study showed that a mass is not dynamically generated in the $\mathcal{N}=2$ model. While we have been able to show that there is strong evidence against the dynamical generation of mass in the three-dimensional extended supersymmetric model, our results depend crucially on the existence of the scale $\bar{\alpha}$ (arising from the size of the compactified dimension). In the fourdimensional model, where this scale is absent, the arguments of Refs. $[9,10]$ could still hold, and the nonrenormalization theorem of Ref. [8] be evaded.

\section{ACKNOWLEDGMENTS}

The authors wish to thank G. Diamandis and B. Georgalas for helpful discussions. The work of N.E.M. was partially supported by P.P.A.R.C. (U.K.). A.C.-S. would like to thank the Theory Division at CERN for their hospitality during the last stages of this work, and gratefully acknowledges financial support from P.P.A.R.C. (U.K.) (grant number 96314661), which has made his visit to CERN possible.

\section{APPENDIX A: SUPERFIELDS IN THREE-DIMENSIONAL $\mathcal{N}=1$ SUPERSPACE.}

Spinor indices are raised and lowered by the antisymmetric metric $C_{\alpha \beta}$, numerically equal to $\sigma_{2}$, such that for any spinor $A$ :

$$
\begin{gathered}
A^{\alpha}=C^{\alpha \beta} A_{\beta}, \\
A_{\alpha}=A^{\beta} C_{\beta \alpha}, \\
C_{\alpha \beta} C^{\mu \nu}=\delta_{[\alpha}^{\mu} \delta_{\beta]}^{\nu}, \\
A^{2} \doteq \frac{1}{2} A^{\alpha} A_{\alpha} \equiv \frac{1}{2} C^{\alpha \beta} A_{\beta} A_{\alpha} .
\end{gathered}
$$

The spinorial derivatives, covariant with respect to supersymmetry, have the following explicit representation:

$$
\begin{aligned}
& D_{\alpha}(x)=\partial_{\alpha}+i \theta^{\beta} \partial_{\alpha \beta}, \\
& D_{\alpha}(p)=\partial_{\alpha}+\theta^{\beta} p_{\alpha \beta},
\end{aligned}
$$

(where $\left\{\partial_{\alpha}, \theta^{\beta}\right\}=\delta_{\alpha}^{\beta}$ ) and they obey the algebra

$$
\left\{D_{\alpha}(p), D_{\beta}(q)\right\}=(p+q)_{\alpha \beta} .
$$

The following identities follow directly from the definition of $D$ :

$$
\begin{gathered}
D^{\alpha}(q) D^{\beta}(q)=q^{\alpha \beta}+C^{\beta \alpha} D^{2}(q), \\
\delta^{2}(12) \delta^{2}(12)=0, \\
\delta^{2}(12) D^{\alpha} \delta^{2}(12)=0, \\
\delta^{2}(12) D^{2} \delta^{2}(12)=\delta^{2}(12), \\
D^{2}(p) \delta^{2}(12) D^{2}(q) \delta^{2}(12)=D^{2}(p+q) \delta^{2}(12) .
\end{gathered}
$$

Scalar superfields have the following component content: scalar, spinor, and auxiliary fields

$$
\begin{gathered}
\phi(x)=\Phi(x, \theta) \mid, \\
\psi_{\alpha}(x)=D_{\alpha} \Phi(x, \theta) \mid, \\
F(x)=D^{2} \Phi(x, \theta) \mid,
\end{gathered}
$$

where the symbol $\mid$ indicates evaluation at $\theta=0$. Equivalently, the component content can be written

$$
\Phi(x, \theta)=\phi(x)+\theta^{\alpha} \psi_{\alpha}(x)-\theta^{2} F(x) .
$$

The components of the connection superfield are given by

$$
\begin{gathered}
u_{\alpha}(x)=\Gamma_{\alpha}(x, \theta) \mid, \\
U(x)=\frac{1}{2} D^{\alpha} \Gamma_{\alpha}(x, \theta) \mid, \\
V_{\alpha \beta}(x)=-\frac{i}{2} D_{(\alpha} \Gamma_{\beta)}(x, \theta) \mid, \\
\lambda_{\alpha}(x)=\frac{1}{2} D^{\beta} D_{\alpha} \Gamma_{\beta}(x, \theta) \mid .
\end{gathered}
$$

The components $u_{\alpha}$ and $U$ can be gauged away in the socalled Wess-Zumino gauge. The remaining physical compo- 

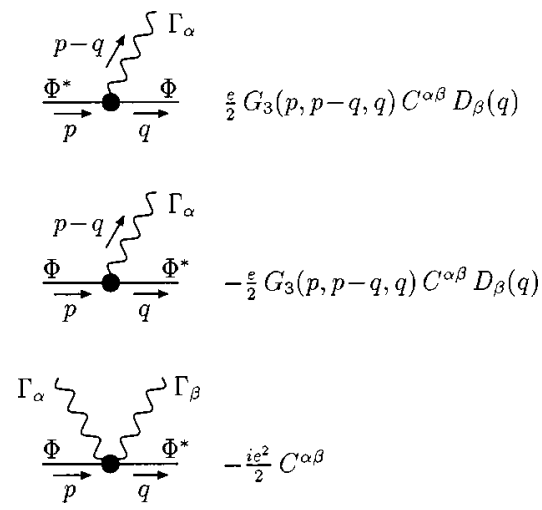

FIG. 4. Interaction vertices in $\mathcal{N}=1$ model.

nents are the normal gauge field $V_{\alpha \beta}$ and its supersymmetric partner $\lambda_{\alpha}$. For further details, the interested reader is referred to Ref. [22]. The superspace Feynman rules from the action in Sec. II are shown in Fig. 4.

\section{APPENDIX B: SUPERFIELDS IN FOUR-DIMENSIONAL $\mathcal{N}=1$ SUPERSPACE}

Spinor indices are raised and lowered by the real metrics $\varepsilon_{\alpha \beta}$ and $\varepsilon_{\alpha \dot{\beta}}$, both numerically equal to the matrix $-i \sigma_{2}$, such that for spinors $\chi$ and $\eta$ :

$$
\begin{array}{ll}
\chi^{\alpha}=\varepsilon^{\alpha \beta} \chi_{\beta}, & \bar{\chi}_{\dot{\alpha}}=\varepsilon_{\alpha \dot{\beta}} \bar{\chi}^{\dot{\beta}}, \\
\chi \eta \doteq \chi^{\alpha} \eta_{\alpha}, & \bar{\chi} \bar{\eta} \doteq \bar{\chi}_{\dot{\alpha}} \bar{\eta}^{\alpha}
\end{array}
$$

The spinorial derivatives have the explicit representation

$$
\begin{gathered}
D_{\alpha}=\partial_{\alpha}+i\left(\sigma^{k} \bar{\theta}\right)_{\alpha} \partial_{k}, \quad \sigma^{k} \rightarrow(\mathbf{1}, \vec{\sigma}), \\
\bar{D}_{\alpha}=-\bar{\partial}_{\alpha}-i\left(\theta \sigma^{k}\right)_{\alpha} \partial_{k},
\end{gathered}
$$

where $\left\{\partial_{\alpha}, \theta^{\beta}\right\}=\delta_{\alpha}^{\beta}$ and $\left\{\bar{\partial}_{\dot{\alpha}}, \bar{\theta}^{\dot{\beta}}\right\}=\delta_{\dot{\alpha}}^{\dot{\beta}}$. The covariant derivatives obey the algebra

$$
\left\{D_{\alpha}(p), \bar{D}_{\alpha}(q)\right\}=\left(\sigma^{k}\right)_{\alpha \dot{\alpha}}(p+q)_{k}
$$

The following identities follow directly from their definition

$$
\begin{gathered}
\delta^{2}(\overline{1} \overline{2}) \delta^{4}(12)=\delta^{2}(12) \delta^{4}(12)=0, \\
D^{2}(q) \delta^{4}(12)=-4 e^{\left(\theta_{1}-\theta_{2}\right) \bar{q}_{1}} \delta^{2}(\overline{1} \overline{2}), \\
\bar{D}^{2}(q) \delta^{4}(12)=-4 e^{\theta_{1} q\left(\bar{\theta}_{1}-\bar{\theta}_{2}\right)} \delta^{2}\left(\theta_{1}-\theta_{2}\right) .
\end{gathered}
$$

Chiral superfields have the following component content (where now the symbol $\mid$ now indicates evaluation at $\theta=\bar{\theta}$ $=0)$ :

$$
\begin{gathered}
\phi(x)=\Phi(x, \theta, \bar{\theta}) \mid, \\
\sqrt{2} \psi_{\alpha}(x)=D_{\alpha} \Phi(x, \theta, \bar{\theta}) \mid, \\
F(x)=-\frac{1}{4} D^{2} \Phi(x, \theta, \bar{\theta}) \mid,
\end{gathered}
$$

which is equivalent to

$$
\Phi(x, \theta, \bar{\theta})=e^{-i \theta \theta \bar{\theta}} \Psi(x, \theta),
$$

$$
\Psi(x, \theta)=\phi(x)+\sqrt{ } 2 \theta \psi(x)+\theta^{2} F(x) .
$$

The component content for an antichiral superfield is similar, with $\theta$ replaced by $\bar{\theta}$, and $\bar{D}$ replacing $D$. The component content of the gauge superfield is as follows:

$$
C(x)=V(x, \theta, \bar{\theta}) \mid,
$$

$$
\chi_{\alpha}(x)=-i D_{\alpha} V(x, \theta, \bar{\theta})\left|, \quad \bar{\chi}_{\dot{\alpha}}(x)=\bar{D}_{\alpha} V(x, \theta, \bar{\theta})\right|,
$$

$$
[M(x)+i N(x)]=\frac{i}{2} D^{2} V(x, \theta, \bar{\theta}) \mid,
$$

$$
\begin{gathered}
{[M(x)-i N(x)]=-\frac{i}{2} \bar{D}^{2} V(x, \theta, \bar{\theta}) \mid,} \\
\lambda_{\alpha}(x)=-\frac{i}{4} \bar{D}^{2} D_{\alpha} V(x, \theta, \bar{\theta})\left|, \quad \bar{\lambda}_{\alpha}(x)=\frac{i}{4} D^{2} \bar{D}_{\alpha} V(x, \theta, \bar{\theta})\right|,
\end{gathered}
$$

$$
\begin{gathered}
\left(\sigma^{k}\right)_{\alpha \alpha} \mathrm{v}_{k}(x)=-\frac{1}{2}\left[D_{\alpha}, \bar{D}_{\alpha}\right] V(x, \theta, \bar{\theta}) \mid, \\
d(x)=\frac{1}{8} D^{\alpha} \bar{D}^{2} D_{\alpha} V(x, \theta, \bar{\theta}) \mid
\end{gathered}
$$

$$
4 \delta_{\alpha}^{\beta} d(x)-2 i\left(\sigma^{m} \bar{\sigma}^{n}\right)_{\alpha}^{\beta} \partial_{[m} \mathrm{v}_{n]}(x)=D^{\beta} \bar{D}^{2} D_{\beta} V(x, \theta, \bar{\theta}) \mid .
$$

In Wess-Zumino gauge the components $C, \chi, \bar{\chi}, M$, and $N$ can all be gauged away, leaving the component expansion

$$
\begin{aligned}
V(x, \theta, \bar{\theta})= & -\left(\theta \sigma^{k} \bar{\theta}\right) \mathrm{v}_{k}(x)+i \theta^{2} \bar{\theta} \bar{\lambda}(x)-\bar{\theta}^{2} \theta \lambda(x) \\
& +\frac{1}{2} \theta^{2} \bar{\theta}^{2} d(x), \\
V^{2}(x, \theta, \bar{\theta})= & -\frac{1}{2} \theta^{2} \bar{\theta}^{2} \mathrm{v}^{2}(x), \\
V^{3}(x, \theta, \bar{\theta})= & 0 .
\end{aligned}
$$

For further details the interested reader is referred to Ref. [25]. The Feynmann rules are very simple; outside the Wess- 
Zumino gauge there are an infinite number of possible vertices, with two external matter superfield lines (one chiral and one antichiral), and $n$ gauge superfields, the vertex corresponding to a factor of $i(2 e)^{n}\left[\right.$ or $i(-2 e)^{n}$ for the fields
$\Phi_{-}$and $\Phi_{-}^{\dagger}$, which are negatively charged under U(1)]. In Wess-Zumino gauge, the series terminates at $n=2$, and we dress the corresponding vertices with the functions $G_{3}$ and $G_{4}$.
[1] R. Pisarski, Phys. Rev. D 29, 2423 (1984).

[2] T. Appelquist, M. Bowick, D. Karabali, and L. Wijewardhana, Phys. Rev. D 33, 3704 (1986).

[3] K. Kondo and H. Nakatani, Prog. Theor. Phys. 87, 193 (1992).

[4] N. Dorey and N.E. Mavromatos, Nucl. Phys. B386, 614 (1992).

[5] P. Maris, Phys. Rev. D 54, 4049 (1996).

[6] I. Aitchison and N.E. Mavromatos, Phys. Rev. B 53, 9321 (1996).

[7] I. Aitchison, G. Amelino-Camelia, M. Klein Kreisler, N.E. Mavromatos, and D. McNeill, Phys. Rev. B 56, 2836 (1997).

[8] T. Clark and S. Love, Nucl. Phys. B310, 371 (1988).

[9] A. Kaiser and S. Selipsky, hep-th/9708087.

[10] T. Appelquist, A. Nyffeler, and S. Selipsky, Phys. Lett. B 425 , 300 (1998).

[11] M. Walker and C. Burden, Phys. Rev. D 59, 125013 (1999).

[12] M. Koopmans and J. Steringa, Phys. Lett. B 226, 309 (1989).

[13] E. Witten, Nucl. Phys. B202, 253 (1982).

[14] M. Walker and C. Burden, Phys. Rev. D (to be published).

[15] T. Clark, O. Piguet, and K. Sibold, Ann. Phys. (N.Y.) 109, 418
(1977).

[16] T. Appelquist and U. Heinz, Phys. Rev. D 24, 2169 (1981).

[17] I. Aitchison, N.E. Mavromatos, and D. McNeill, Phys. Lett. B 402, 154 (1997).

[18] K. Kondo and T. Murakami, Phys. Lett. B 410, 257 (1997).

[19] O. Alvarez, Phys. Rev. D 17, 1123 (1978).

[20] M. Ciuchini and J. Gracey, Nucl. Phys. B454, 103 (1995).

[21] G. Diamandis, B. Georgalas, and N.E. Mavromatos, Mod. Phys. Lett. A 13, 387 (1998).

[22] S. Gates, M. Grisaru, M. Roček, and W. Siegel, Superspace: One Thousand And One Lessons In Supersymmetry, Vol. 58 of Frontiers in Physics (Benjamin-Cummings, Reading, MA, 1983).

[23] D. Curtis and M. Pennington, Phys. Rev. D 42, 4165 (1990).

[24] A. Campbell-Smith, Mod. Phys. Lett. A 13, 2337 (1998).

[25] P. Srivastava, Supersymmetry, Superfields and Supergravity: An Introduction (Adam Hilger, Bristol, 1986).

[26] R. Pisarski, Phys. Rev. D 44, 1866 (1991).

[27] M. Pennington and D. Walsh, Phys. Lett. B 253, 245 (1991). 